Provided for non-commercial research and educational use only. Not for reproduction or distribution or commercial use.

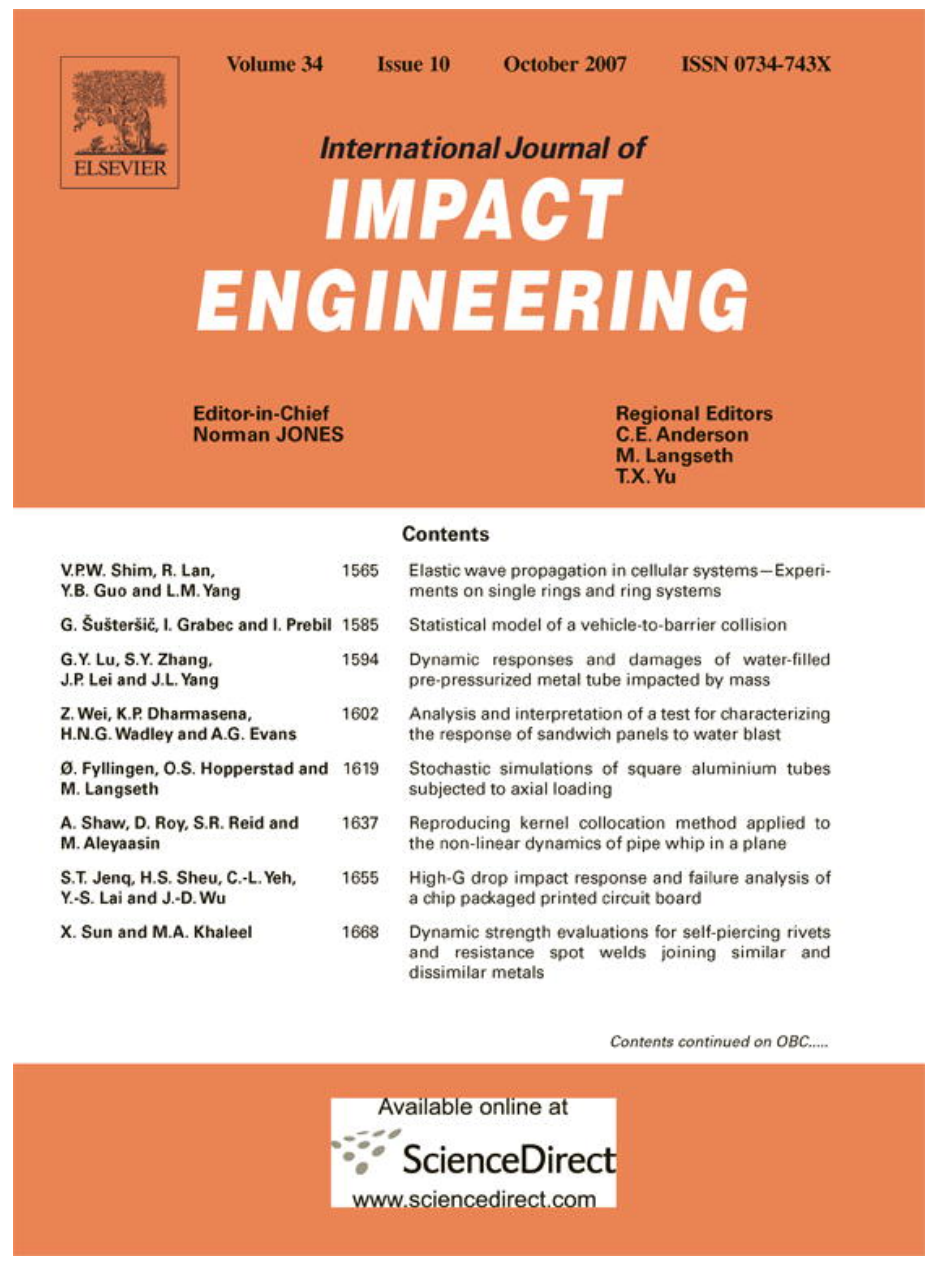

This article was originally published in a journal published by Elsevier, and the attached copy is provided by Elsevier for the author's benefit and for the benefit of the author's institution, for non-commercial research and educational use including without limitation use in instruction at your institution, sending it to specific colleagues that you know, and providing a copy to your institution's administrator.

All other uses, reproduction and distribution, including without limitation commercial reprints, selling or licensing copies or access,

or posting on open internet sites, your personal or institution's website or repository, are prohibited. For exceptions, permission may be sought for such use through Elsevier's permissions site at:

http://www.elsevier.com/locate/permissionusematerial 


\title{
Analytical calculation of blast-induced strains to buried pipelines
}

\author{
George P. Kouretzis ${ }^{\mathrm{a}}$, George D. Bouckovalas ${ }^{\mathrm{a}, *}$, Charis J. Gantes ${ }^{\mathrm{b}}$ \\ ${ }^{a}$ Department of Geotechnical Engineering, Faculty of Civil Engineering, National Technical University of Athens, 9, Iroon Polytehniou Street \\ GR-157 80, Athens, Greece \\ ${ }^{\mathrm{b}}$ Department of Structural Engineering, Faculty of Civil Engineering, National Technical University of Athens, 9, Iroon Polytehniou Street \\ GR-157 80, Athens, Greece
}

Received 24 August 2005; received in revised form 14 May 2006; accepted 30 August 2006

Available online 15 November 2006

\begin{abstract}
The aim of this paper is to introduce a robust methodology for the analytical calculation of strains in flexible buried pipelines due to surface point-source blasts. Following a brief bibliographic overview regarding the characteristics of ground waves produced by surface explosions, a method used to model wave propagation with radial attenuation and spherical front is presented. Strains due to P- and Rayleigh waves, which dominate the waveform generated by an explosion, are accordingly calculated by modeling the pipeline as a three-dimensional (3-D) cylindrical thin shell and ignoring soil-structure interaction. To simplify the design procedure, a set of easy-to-use relations for the calculation of maximum strains and their position along the pipeline axis is supplied. The derived expressions are evaluated through comparison against 3-D dynamic numerical analyses, field strain measurements in flexible pipelines due to a series of full scale blasts, and state-of-practice design methods. Comparisons show that the proposed methodology provides improved accuracy at no major expense of simplicity, as well as the advantage of properly accounting for the effect of local soil conditions.
\end{abstract}

(C) 2006 Elsevier Ltd. All rights reserved.

Keywords: Blast; Buried pipelines; Shell theory; Design; Safety distance

\section{Introduction}

Despite the remarkable expansion in the use of buried pipeline networks over the last few years, limited fresh literature refers to their design against ground shock wave propagation. In fact, seismic design of buried pipelines is still based on the expressions proposed by Newmark [1] and Kuesel [2]. This is due to the fact that earthquake induced ground motion is rarely strong enough to affect steel pipelines, as proven by their in situ response in various earthquakes. Nevertheless, accidental or intended surface explosions (e.g. an accident in an explosive storage facility or routine quarry blasts) may generate ground waves with significant amplitude in short distance from the source of the explosion, much larger than that originating from a strong earthquake, and prove threatening for the pipeline. The precise calculation of the safety distance from the source of a

*Corresponding author. Tel.: + 302107723780 ; fax: + 302107723428 .
E-mail address: gbouck@central.ntua.gr (G.D. Bouckovalas). 
potentially threatening explosion could be of outmost importance for the rational design of a pipeline, as overconservative estimates can lead to unnecessary re-routing, and to a disproportionate increase in the cost of the project.

The design of pipelines against blasts is based today either on analytical relations originally proposed for seismic waves with plane front and constant amplitude [3] or by an empirical relation initially proposed by Esparza et al. [4] and lately embraced by the ASCE-ALA guidelines [5]. It is realized that the first approach is a rather crude approximation, which was merely employed to provide conservative estimates in the absence of any problem-specific solutions. On the contrary, the later approach has been developed specifically for the problem at hand, based on an experimental database for blast-induced strains on instrumented pipe segments. Nevertheless, this approach is purely empirical, and consequently its range of application should be limited to conditions similar to the conditions prevailing in the relevant experiments.

This paper aims at filling this gap in the modern literature by presenting a new analytical methodology for calculating strains in buried pipelines due to surface point-source blasts. The proposed methodology incorporates three-dimensional (3-D) thin shell theory for the accurate modeling of the pipeline response and properly accounts for the spherical front and the radial attenuation of ground vibration induced by blast waves. The methodology is mainly intended for the design of buried steel pipelines, but its assumptions are also valid for flexible pipelines made of other materials such as PVC.

It is acknowledged that the theoretical derivation of pipeline strains with the proposed methodology is rather complex, and difficult to follow during this, necessarily concise, presentation. For this reason, emphasis is placed upon the basic assumptions of the methodology, while the analytical results are evaluated with the aid of 3-D dynamic numerical simulations of the pipeline response, and compared to field strain measurements in flexible pipelines due to a series of full-scale blasts [6]. Furthermore, easy-to-use design relations are established for use in practical applications.

\section{Ground shock waves induced by a surface explosion}

In a simple way, surface point-source blasts may be simulated as a time-dependent vertical point load acting on the surface of a homogeneous, isotropic, elastic half space (Fig. 1a). This is commonly referred as Lamb's problem, after the name of the researcher who first formulated it and solved it [7]. Keeping in mind that the
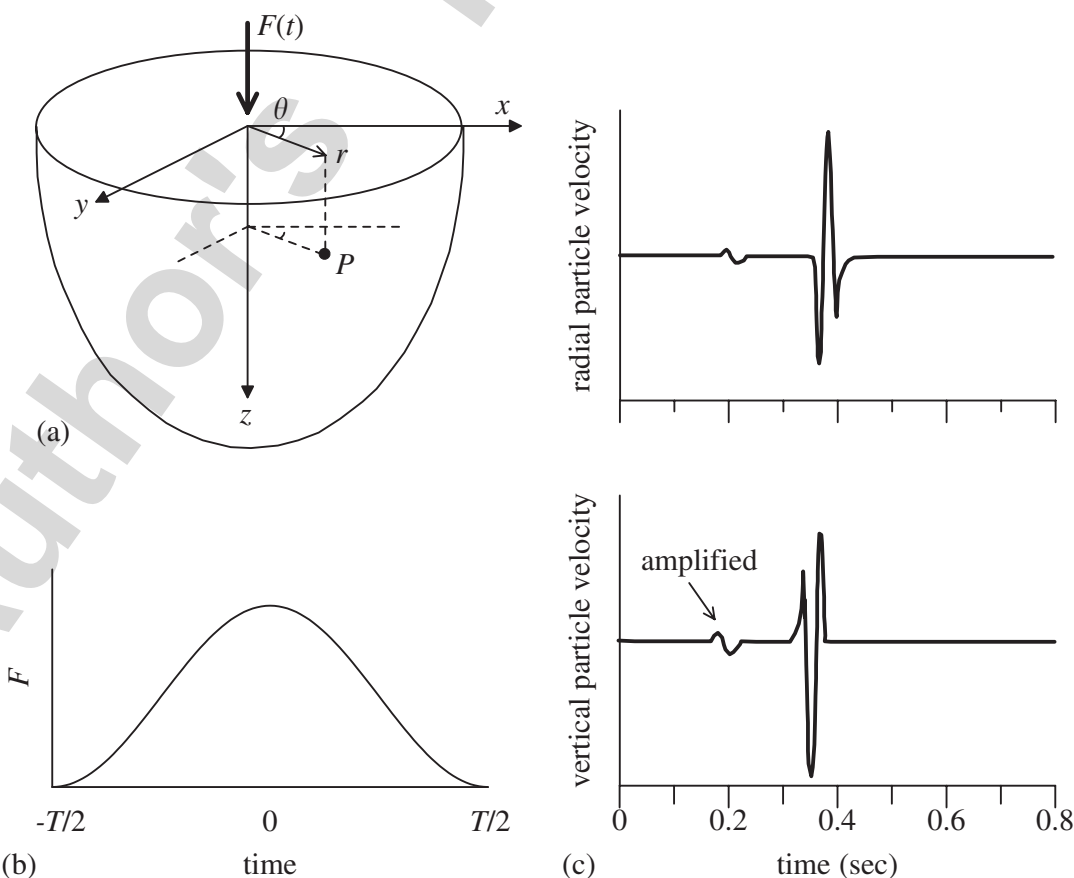

Fig. 1. (a) Definition of the cylindrical coordinate system used in the analytical evaluation of the response of an elastic half-space to the concentrated dynamic load shown in (b). Typical resulting peak particle velocity time histories are illustrated in (c) (after Mooney [8]). 
load, as well as the geometry, is vertically axisymmetric, the transverse component of ground displacement is neglected and the dynamic equations of motion are solved in the two-dimensional (2-D) space, i.e. in the vertical plane through the center of the explosion.

Mooney [8] was among the first researchers to provide a series of closed-form solutions for the calculation of the displacements and velocities resulting from the application of a bell-shaped load pulse, described by Eq. (1) (Fig. 1b):

$$
F(t)=F \cos ^{2}\left(\frac{\pi t}{T}\right) \quad \text { where }-T / 2 \leqslant t \leqslant T / 2,
$$

where $F$ and $T$ are the amplitude and the total duration of the pulse, respectively, while $t$ stands for time.

Mooney's predictions show that both the radial as well as the vertical component of particle velocity attenuate exponentially with the distance from the source. The relation between the peak particle velocity $V_{\max }$ and the distance $R$ can be written under the general form [8]

$$
V_{\max }=E R^{-n} \text {. }
$$

Constant $E$ is a function of the load and the medium characteristics, while the attenuation exponent $n$ depends on the properties of the half-space as well as the radial distance. Namely, $n$ in the near field obtains values between 1.27 and 0.84 , and is further reduced between 0.72 and 0.42 at larger radial distances. The predicted response in the near field reflects the attenuation of compressional (P) waves, while in the far field it reflects the slower attenuation of surface Rayleigh $(\mathrm{R})$ waves.

Fig. 1c illustrates two typical particle velocity time histories, one for the radial and the other for the vertical component of the ground motion, obtained from the analytical solution of Mooney [8]. It should be noted that both time histories refer to the far-field, a fact that justifies the rather long time lag between the arrival of the two waveforms, and the small amplitude of the P-wave with respect to the amplitude of the Rayleigh wave. By examining both waveforms, it is observed that:

(a) as expected, the arrival of $\mathrm{P}$ waves precedes the arrival of Rayleigh waves,

(b) the amplitude of the vertical component of the $\mathrm{P}$ wave is negligible compared to the amplitude of its radial component,

(c) the arrival of Rayleigh waves is signaled by a smooth positive (upward) change of the vertical velocity and a steep negative (downward) change in the radial velocity,

(d) S-waves are overshadowed by the practically concurrent arrival of Rayleigh waves with larger amplitude.

It should be reminded here that all the above are valid for an elastic medium. In fact, actual soil behavior is elasto-plastic and its hysteretic damping cannot be considered in an analytical solution. Non-linear dynamic numerical analyses [9] verify that the value of the attenuation exponent is associated with the propagation of $\mathrm{P}$ waves in the near field and Rayleigh waves in larger distances, as suggested by the analytical solution. However, the numerically predicted attenuation rates depend on the soil characteristics, and are much larger than the analytically predicted ones $[9,10]$.

A number of empirical attenuation relations in the general form of Eq. (2) have been proposed in the literature for the approximate calculation of the peak particle velocity. However, most of them concern deep underground explosions at purely rock sites, and are not directly applicable to the problem studied herein. Only a few publications (e.g. [6,11-13]) refer to wave propagation from surface or near-surface blasts and propose relations which are applicable to soil and rock formations alike.

\section{Methodology outline and assumptions}

As mentioned above, the proposed methodology applies to long and flexible cylindrical pipelines. In more detail, the following assumptions are adopted with regard to the pipeline behavior:

(a) The inertia and kinematic interaction effects between the buried pipeline and the surrounding soil can be ignored. Theoretical arguments and numerical simulations plead for the general validity of the former 
statement regarding inertia effects [14], while the importance of kinematic interaction effects can be checked on a case-by-case basis via the flexibility index:

$$
F=\frac{2 E_{\mathrm{m}}\left(1-v_{1}^{2}\right)(D / 2)^{3}}{E_{1}\left(1+v_{\mathrm{m}}\right) t_{\mathrm{s}}^{3}},
$$

where $E_{\mathrm{m}}$ is the Young's modulus of the surrounding soil, $E_{1}$ the Young's modulus of the pipe material, $v_{\mathrm{m}}$ the Poisson's ratio of the surrounding soil, $v_{1}$ the Poisson's ratio of the pipe material, $t_{\mathrm{s}}$ the thickness of the cross-section, and $D$ the pipe diameter.

The flexibility index is related to the ability of the lining to resist distortion from the ground [15]. Values of the flexibility index higher than 20 are obtained for most common pipelines, indicating that ignoring overall the soil-structure interaction is a sound engineering approach $[6,16,17]$. Moreover, keeping in mind that, even in very stiff soil or rock formations, the wavelength of blast-induced strong motion does not exceed $10 \mathrm{~m}$ [3], i.e. it is much smaller than typical pipeline diameters, wave scattering effects in the soil-pipeline boundary are considered negligible, and are ignored herein. As a result from the above, the pipeline here is assumed to fully conform to the ground motion, and its displacements are considered equal to those of the surrounding soil. The validity of this assumption is also verified experimentally via blastinduced displacement time history measurements on flexible pipelines and in the surrounding soil, presented by Siskind et al. [6].

(b) The pipe is modeled as a 3-D thin elastic shell (Fig. 2), where normal (axial $\varepsilon_{\mathrm{a}}$ and hoop $\varepsilon_{\mathrm{h}}$ ) as well as shear $(\gamma)$ strains develop along the axis and the perimeter, but not along the radius of the pipeline [18]. Not accounting for material non-linearity is common in such analytical solutions, as steel pipelines constructed with in situ welds are considered to fail at their joints for relatively low strain levels (i.e. $0.5 \%$ ), well below the yield limit of the pipeline material.

(c) There is no slippage at the soil-pipe interface. It can be shown [19] that this is a conservative assumption as it leads to larger overall strains compared to the condition of a "smooth" interface, where the pipeline is free to slip relatively to the surrounding soil. In addition, the presence of the cavity formed in the ground by the construction of the pipeline is also ignored, thus a non-perforated ground model is adopted for the calculation of pipeline strains due to transversely propagating waves. Although this assumption does not affect the calculation of axial strains, it provides upper bound estimates of hoop strains for a wide range of pipelines with flexibility ratios $20<F<10,000$.

As structural inertia is neglected, the number of significant circles of the excitation does not affect the response of the pipeline. Thus, the pulse-like ground motion induced by the blast can be replaced by a train of harmonic waves with constant amplitude, which is simpler to treat analytically by solving the equivalent steady-state problem.

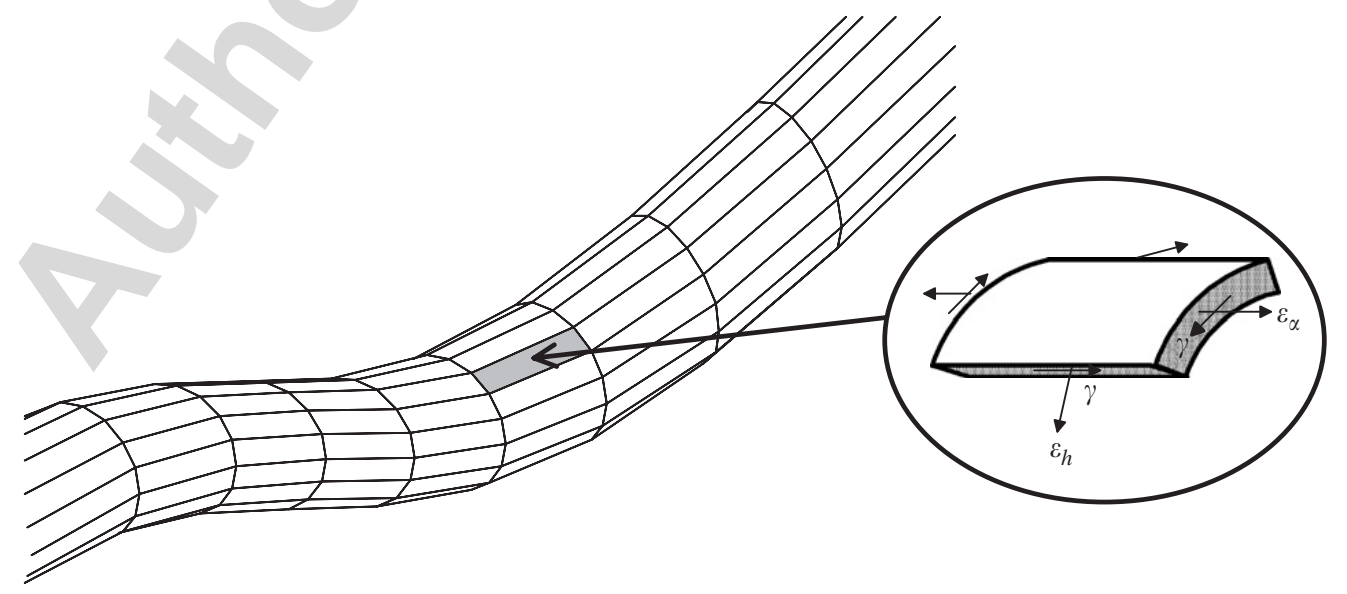

Fig. 2. Strain notation for thin-walled cylindrical shells. 
Ground waves are assumed to propagate from the center of the point-source explosion with a spherical wave front. The attenuation of the peak particle velocity with the distance from the source, regardless of the wave type that it is attributed to, is quantitatively described by Eq. (2). For harmonic waves, Eq. (2) can be rewritten in terms of displacement as

$$
A_{\max }=A(R / d)^{-n}
$$

where

$$
A=(E / 2 \pi v) d^{-n}
$$

is the maximum ground displacement at the projection of the explosion source on the pipe (Point O in Fig. 3), $v$ is the frequency of the harmonic wave, $d$ is the distance of the explosion source from the pipe axis (Fig. 3), and

$$
R=\sqrt{z^{2}+d^{2}}
$$

If the elevation of the pipeline axis relatively to the source of the explosion is equal to $H$, distance $d$ is expressed as

$$
d=\sqrt{d_{\mathrm{h}}^{2}+H^{2}},
$$

where $d_{\mathrm{h}}$ is the horizontal distance between the pipeline and the point source. However, for the case of nearsurface blasts examined here, $H$ is considered much smaller than $d_{\mathrm{h}}$ and will be neglected hereafter.

In the following sections, strains on the pipeline are computed analytically by considering the action of Pand Rayleigh waves, that appear to dominate the response in small and larger distances from the source of the explosion, respectively $[8,9]$.

\section{Strain analysis for $P$ waves}

Consider a harmonic $\mathrm{P}$ wave propagating with spherical front in the horizontal plane and a pipeline constructed at horizontal distance $d$ from the center of the explosion (Fig. 3). Referring to a polar coordinate system originating from the center of the explosion, the corresponding ground motion can be expressed analytically as

$$
u_{R}=A_{\max } \sin \left[\frac{2 \pi}{L}\left(R-C_{\mathrm{p}} t\right)\right]
$$

where $C_{\mathrm{p}}$ is the propagation velocity of $\mathrm{P}$ waves, $L=C_{\mathrm{p}} T$ the wavelength, $T$ the wave period, $A_{\max }$ the maximum ground displacement at radial distance $R$ from the source, and $t$ stands for time.

To aid the analytical computation of strains, ground displacement is vectorially decomposed into two components (Fig. 3): one with motion parallel to the undeformed pipe axis and the other with motion perpendicular to it. As the deformation of the surrounding soil is transferred unaltered to the buried structure, the displacements induced on each point of a 3-D cylindrical shell with radius $r$ equal to the radius of the pipeline will be

$$
\begin{aligned}
& u_{x}=A_{\max } \cos \omega \sin \left[\frac{2 \pi}{L}\left(R^{\prime}-C_{\mathrm{p}} t\right)\right], \\
& u_{y}=0, \\
& u_{z}=A_{\max } \sin \omega \sin \left[\frac{2 \pi}{L}\left(R^{\prime}-C_{\mathrm{p}} t\right)\right],
\end{aligned}
$$

where

$$
\omega=\arctan \left(\frac{z}{d+r \sin \theta}\right) \text { and } \quad R^{\prime}=\sqrt{z^{2}+(d+r \sin \theta)^{2}} .
$$




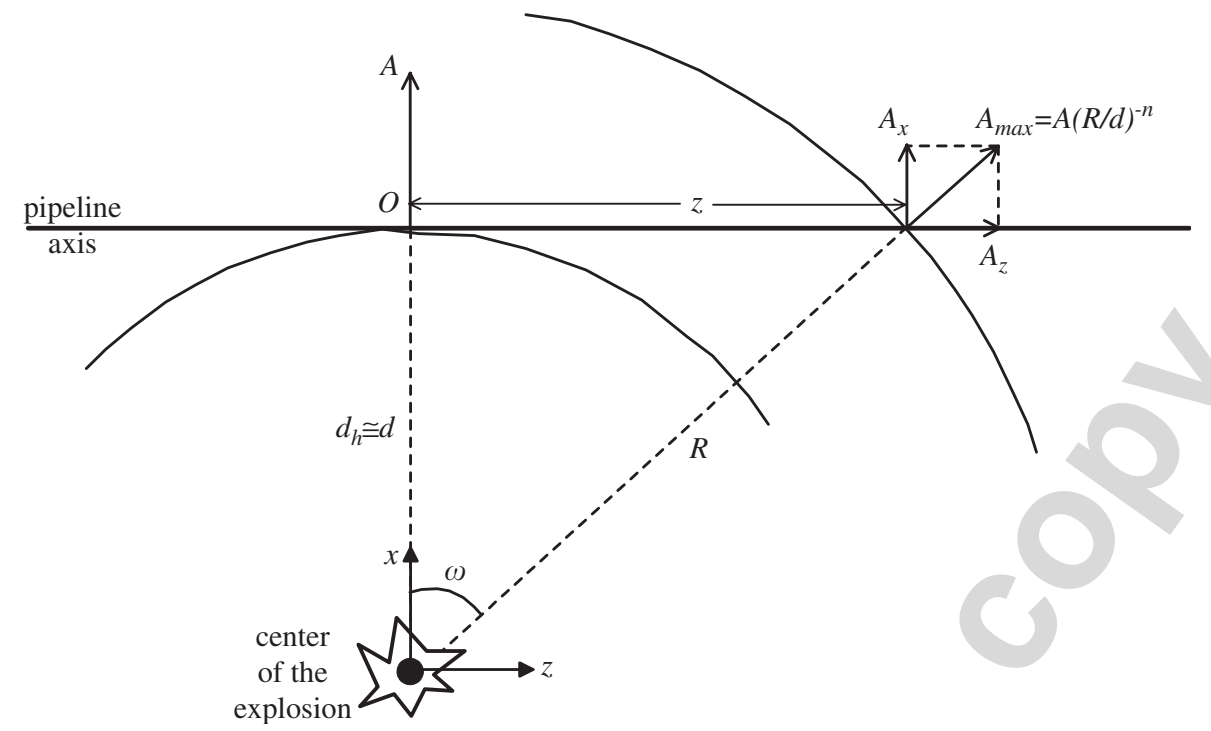

Fig. 3. Definition of the peak particle displacement $A_{\max }$ applied to the pipeline, as a result of the radial propagation of a blast-induced P wave in the horizontal plane.
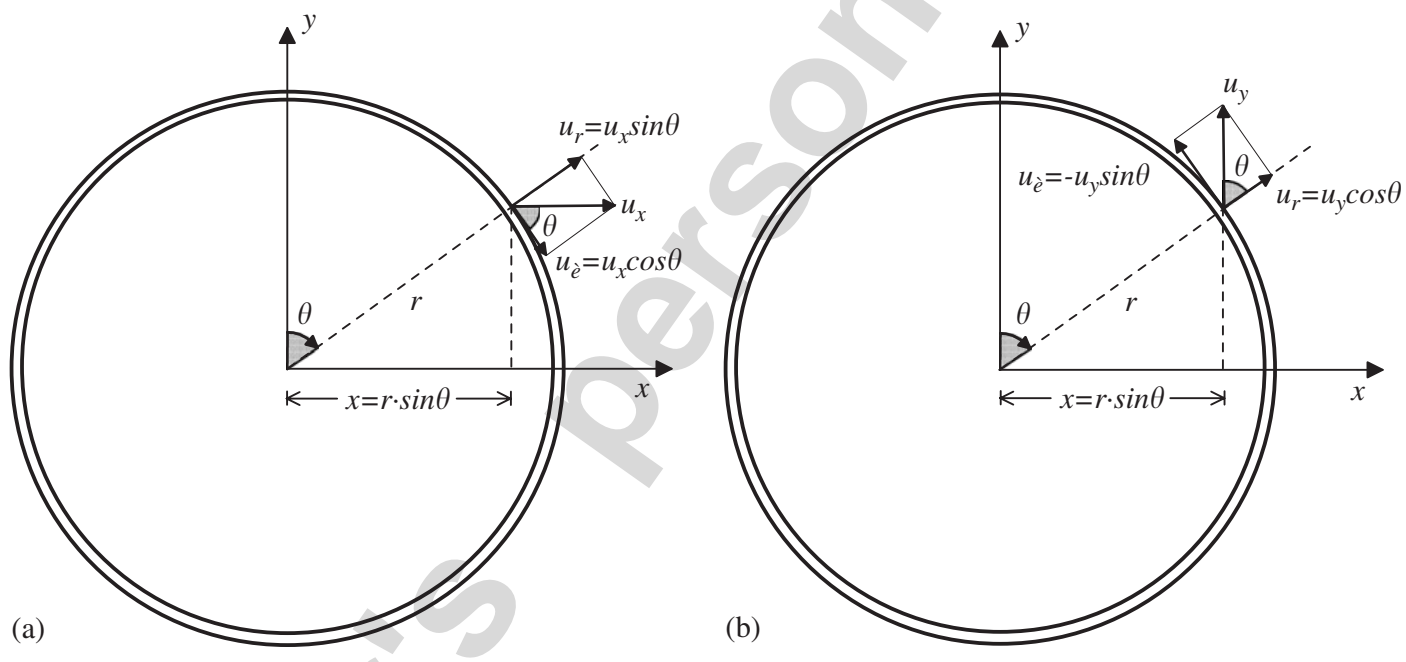

Fig. 4. The cylindrical coordinate system employed in the strain analysis of the 3-D shell due to the propagation (a) of a P wave and (b) of the SV component of a Rayleigh wave.

In Eq. (12), $z$ is the distance of any given cross-section from the projection of the center of the explosion on the pipe axis (Point $\mathrm{O}$ in Fig. 3) and $r, \theta$ define the location of each point on the shell in cylindrical coordinates (Fig. 4a).

Strains on the 3-D shell may be computed separately for each one of the components of the enforced displacement and consequently superimposed, as follows.

Strains due to the perpendicular to the pipeline axis component of displacement, $u_{x}$ : In a cylindrical coordinate system fitted to the axis of the structure (Fig. 4a), the imposed displacement $u_{x}$ can be decomposed into the following radial and tangential components:

$$
\begin{aligned}
& u_{r}=A\left(\frac{R}{d+r \sin \theta}\right)^{-n} \sin \theta \cos \omega \sin \left[\frac{2 \pi}{L}\left(R^{\prime}-C_{\mathrm{p}} t\right)\right], \\
& u_{\theta}=A\left(\frac{R}{d+r \sin \theta}\right)^{-n} \cos \theta \cos \omega \sin \left[\frac{2 \pi}{L}\left(R^{\prime}-C_{\mathrm{p}} t\right)\right] .
\end{aligned}
$$


According to the "elastic thin shell" assumption adopted herein [18], the corresponding strains in the structure are calculated from the stress-displacement equations in cylindrical coordinates as

$$
\begin{aligned}
& \varepsilon_{\alpha}= \varepsilon_{z z}=\frac{\partial u_{z}}{\partial z}=0 \\
& \varepsilon_{\mathrm{h}}= \varepsilon_{\theta \theta}=\frac{1}{r} \frac{\partial u_{\theta}}{\partial \theta}+\frac{u_{r}}{r}=\frac{A}{L R^{3}}(R / d)^{-n} \\
& \times \cos ^{2} \theta\left(2 d^{2} \pi R \cos \left[\frac{2 \pi}{L}\left(R-C_{\mathrm{p}} t\right)\right]-L z^{2}(1+n) \sin \left[\frac{2 \pi}{L}\left(R-C_{\mathrm{p}} t\right)\right]\right), \\
& \gamma=\gamma_{\theta z}= \frac{1}{r} \frac{\partial u_{z}}{\partial \theta}+\frac{\partial u_{\theta}}{\partial z}=\frac{A z d}{L R^{3}}(R / d)^{-n} \\
& \quad \times \cos \theta\left(2 \pi R \cos \left[\frac{2 \pi}{L}\left(R-C_{\mathrm{p}} t\right)\right]-L(1+n) \sin \left[\frac{2 \pi}{L}\left(R-C_{\mathrm{p}} t\right)\right]\right) .
\end{aligned}
$$

The resulting Eqs. (16) and (17) that provide the strains on the shell have been simplified by assuming that the radius of the pipeline $r$ is small compared to the distance from the explosion $d$ :

$$
d+r \sin \theta \approx d \quad \text { or } R^{\prime} \approx R .
$$

Strains due to the parallel to the pipeline axis component of displacement, $u_{z}$ : The harmonic displacement $u_{z}$ induced to the shell (Eq. (11)) leads to the development of the following strains:

$$
\begin{aligned}
& \varepsilon_{\alpha}= \varepsilon_{z z}=\frac{\partial u_{z}}{\partial z}=\frac{A}{L R^{3}}(R / d)^{-n} \\
& \times\left(2 \pi z^{2} R \cos \left[\frac{2 \pi}{L}\left(R-C_{\mathrm{p}} t\right)\right]+L\left(d^{2}-n z^{2}\right) \sin \left[\frac{2 \pi}{L}\left(R-C_{\mathrm{p}} t\right)\right]\right) \\
& \varepsilon_{\mathrm{h}}= \varepsilon_{\theta \theta}=\frac{1}{r} \frac{\partial u_{\theta}}{\partial \theta}+\frac{u_{r}}{r}=0 \\
& \gamma=\gamma_{\theta z}=\frac{1}{r} \frac{\partial u_{z}}{\partial \theta}+\frac{\partial u_{\theta}}{\partial z}=\frac{A z}{d L R^{3}}(R / d)^{-n} \\
& \quad \times \cos \theta\left(2 d^{2} \pi R \cos \left[\frac{2 \pi}{L}\left(R-C_{\mathrm{p}} t\right)\right]+L\left(-d^{2}+n z^{2}\right) \sin \left[\frac{2 \pi}{L}\left(R-C_{\mathrm{p}} t\right)\right]\right) .
\end{aligned}
$$

The shear strain calculated from Eq. (21) is in phase with the shear strain resulting from the $u_{x}$ component of motion (Eq. (17)). Thus, the total shear strain on the cross-section will result from the superposition of the expressions of Eqs. (21) and (17) or

$$
\begin{aligned}
\gamma= & \frac{A z}{d L R^{3}}(R / d)^{-n} \cos \theta\left(4 d^{2} \pi R \cos \left[\frac{2 \pi}{L}\left(R-C_{\mathrm{p}} t\right)\right]\right. \\
& \left.+L\left(-d^{2}(2+n)+n z^{2}\right) \sin \left[\frac{2 \pi}{L}\left(R-C_{\mathrm{p}} t\right)\right]\right) .
\end{aligned}
$$

Comparison with numerical analyses: To check the accuracy of the above analytical calculations, the effect of blast-induced $\mathrm{P}$ waves on a cylindrical thin shell was simulated with a dynamic 3-D FEM analysis, conducted with the commercially available code ANSYS [20]. It is clarified in advance that the aim of this comparison was not to check the validity of the assumptions, but only to check the complex mathematics that underlay the computation of shell strains.

Fig. 5 presents the geometry of the pipeline and the applied ground displacements resulting from the radial propagation of a harmonic $\mathrm{P}$ wave with infinite duration. More specifically, the pipeline is modeled as a thinwalled cylindrical segment of $1 \mathrm{~m}$ external diameter and $20 \mathrm{~mm}$ wall thickness, at $10 \mathrm{~m}$ distance from the center of the explosion. Taking advantage of symmetry with regard to the projection of the center of the explosion on the pipeline axis, only one-half of the pipeline segment is considered for the analysis, with a length of $30 \mathrm{~m}$ 


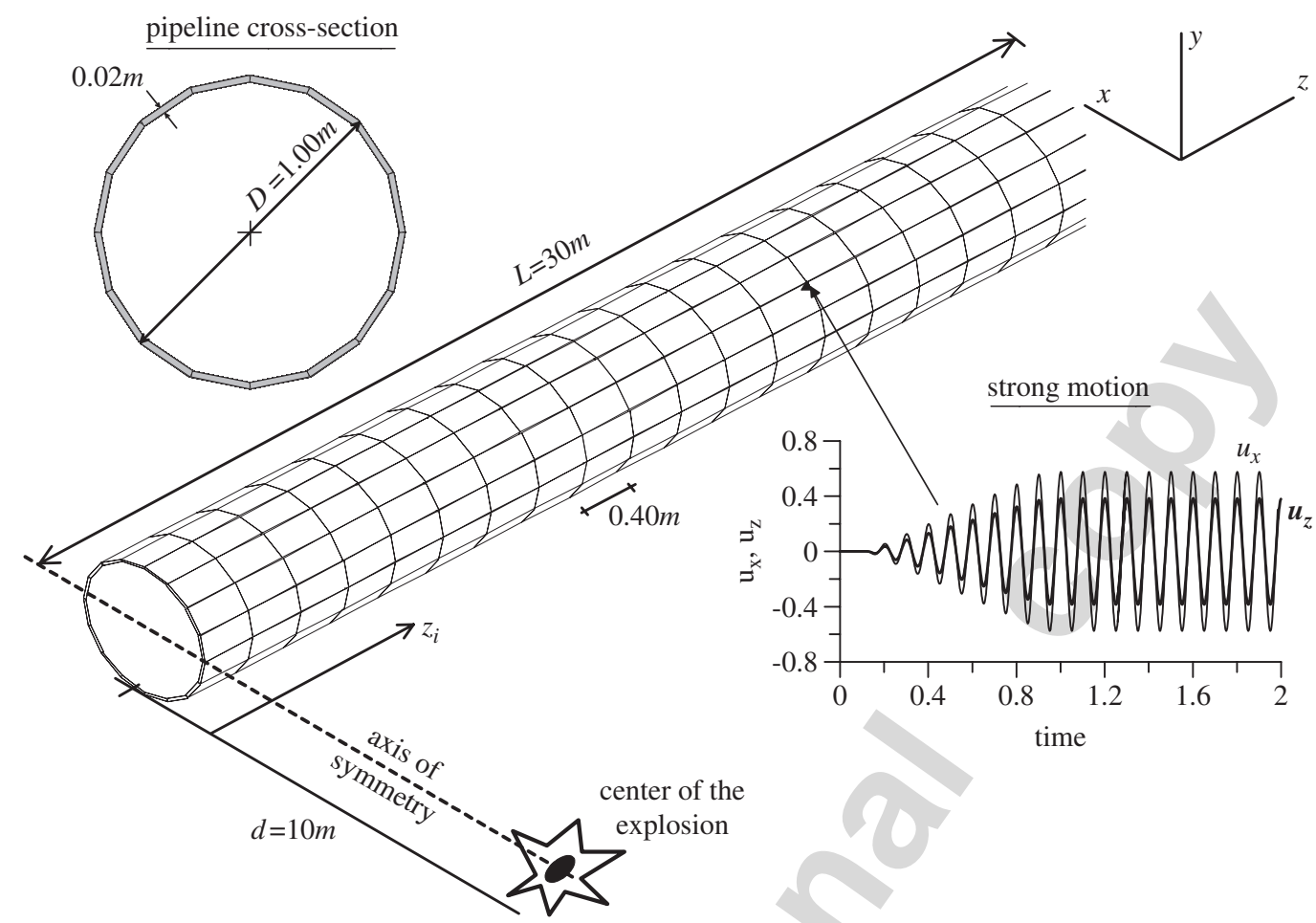

Fig. 5. Mesh discretization of the 3-D numerical model and dynamically imposed displacements on a random node (this figure is out of scale).

(Fig. 5). The cylinder is discretized with 4-node shell elements with membrane and bending capabilities, using 16 equally sized, $0.4 \mathrm{~m}$ long, elements per cross-section. The pipe material is considered to be isotropic, linear elastic with Young's modulus $E_{1}=210,000 \mathrm{MPa}$, specific weight $\gamma_{1}=75 \mathrm{kN} / \mathrm{m}^{3}$ and Poisson's ratio $v_{1}=0.20$. Note that the exact values of $\gamma_{1}, E_{1}$ and $v_{1}$ are of absolutely no importance to the numerical results, as seismic strain components $\varepsilon_{\alpha}, \varepsilon_{\mathrm{h}}$ and $\gamma$ are directly related to the imposed displacements alone (e.g. Eqs. (15)-(17)).

The displacement time history is harmonic, corresponding to a wave with period $T=0.1 \mathrm{~s}$ and propagation velocity $C=100 \mathrm{~m} / \mathrm{s}$. The amplitude of the strong motion attenuates exponentially with the distance from the source according to Eq. (4), with $A=1 \mathrm{~m}$ and $n=2$.

As the pipeline fully conforms to the ground motion, the displacement of each node of the 3-D shell is considered equal to the corresponding ground displacement. Hence, in the Cartesian coordinate system that was adopted for the preceding strain analysis of the pipeline (Fig. 3), the displacement time histories applied at node $i$ are expressed by

$$
\begin{aligned}
& u_{x, i}=A\left(\frac{R_{i}}{x_{i}}\right)^{-n} \sin \omega_{i} \sin \left[\frac{2 \pi}{L}\left(R_{i}-C t\right)\right], \\
& u_{z, i}=A\left(\frac{R_{i}}{x_{i}}\right)^{-n} \cos \omega_{i} \sin \left[\frac{2 \pi}{L}\left(R_{i}-C t\right)\right]
\end{aligned}
$$

and

$$
u_{y, i}=\theta_{x, i}=\theta_{y, i}=\theta_{z, i}=0 .
$$

In the above equations $R_{i}=\sqrt{z_{i}^{2}+x_{i}^{2}}, \omega_{i}=\arctan \left(z_{i} / x_{i}\right)$, while $x_{i}, z_{i}$ are the coordinates of each node at the aforementioned Cartesian system. It must be noted here that the amplitude of the ground motion is gradually increasing with time, and the maximum value is attained after 8 full cycles (Fig. 5). This provision is necessary 

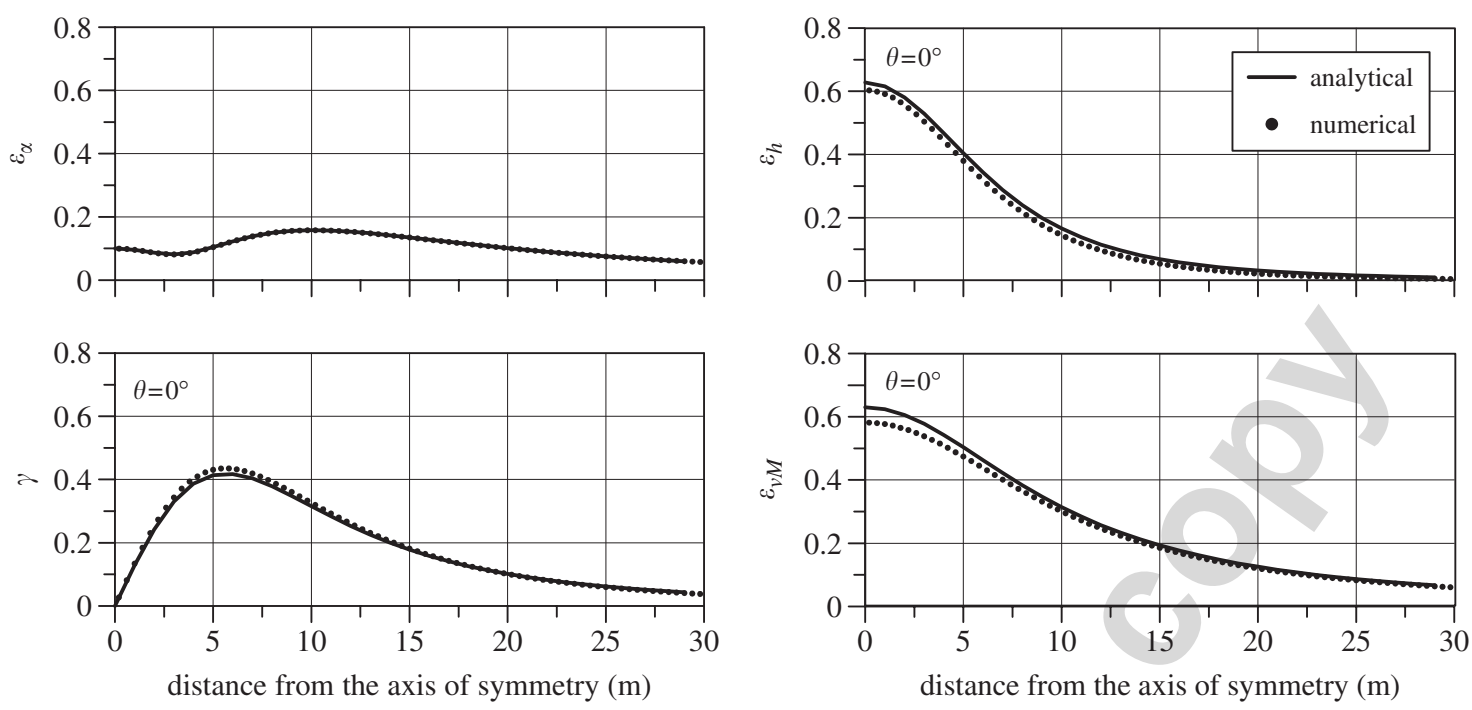

Fig. 6. Comparison of analytical and numerical results along the pipeline axis. The polar angle $\theta$ where each maximum strain appears is also shown (P wave).

in order to avoid numerical pseudo-oscillations, resulting from the sudden application of a uniform amplitude excitation.

Numerically and analytically predicted maximum strains along the pipeline are compared in Fig. 6. The comparison is shown in terms of the maximum overall (i.e. for the entire duration of the motion and all over the cross-section) strain along the pipeline, although strains do not reach their maximum values simultaneously at every cross-section of the shell. It is observed that numerical and analytical predictions are in fairly good agreement for all strain components (Fig. 6).

\section{Strain analysis for Rayleigh waves}

As mentioned earlier in the description of Lamp's problem, Rayleigh waves dominate the strong motion waveform at relatively large distances from the explosion. According to basic wave propagation theory, a Rayleigh wave is equivalent to a $\mathrm{P}$ wave and an SV wave propagating simultaneously along the same path, with velocity $C_{R}$ and a phase difference of $\pi / 2$. Close to the free surface of an elastic half space with Poisson's ratio $v_{\mathrm{m}}=0.25$, the amplitude ratio of these two components can be computed analytically [21], as

$$
\frac{A_{\max , \mathrm{V}}}{A_{\max , \mathrm{H}}}=1.4677=k,
$$

where $A_{\max , \mathrm{V}}$ is the amplitude of the $\mathrm{SV}$ wave component and $A_{\max , \mathrm{H}}$ is the amplitude of the $\mathrm{P}$-wave component. In the following, the presentation focuses upon strains due to the SV wave component, as strains due to the $\mathrm{P}$ wave component have been essentially discussed previously.

The displacement vector of an SV wave lies within the vertical plane defined by the radius of propagation $R$ and the vertical axis $y$ (Fig. 7). The harmonic displacement induced on each point of the 3-D shell representing the pipeline is considered equal to the ground displacement, and can be expressed mathematically as

$$
u_{y}=A_{\max } \sin \left[\frac{2 \pi}{L}\left(R^{\prime}-C_{R} t\right)\right]
$$

where

$$
R^{\prime}=\sqrt{z^{2}+(d+r \sin \theta)^{2}}
$$




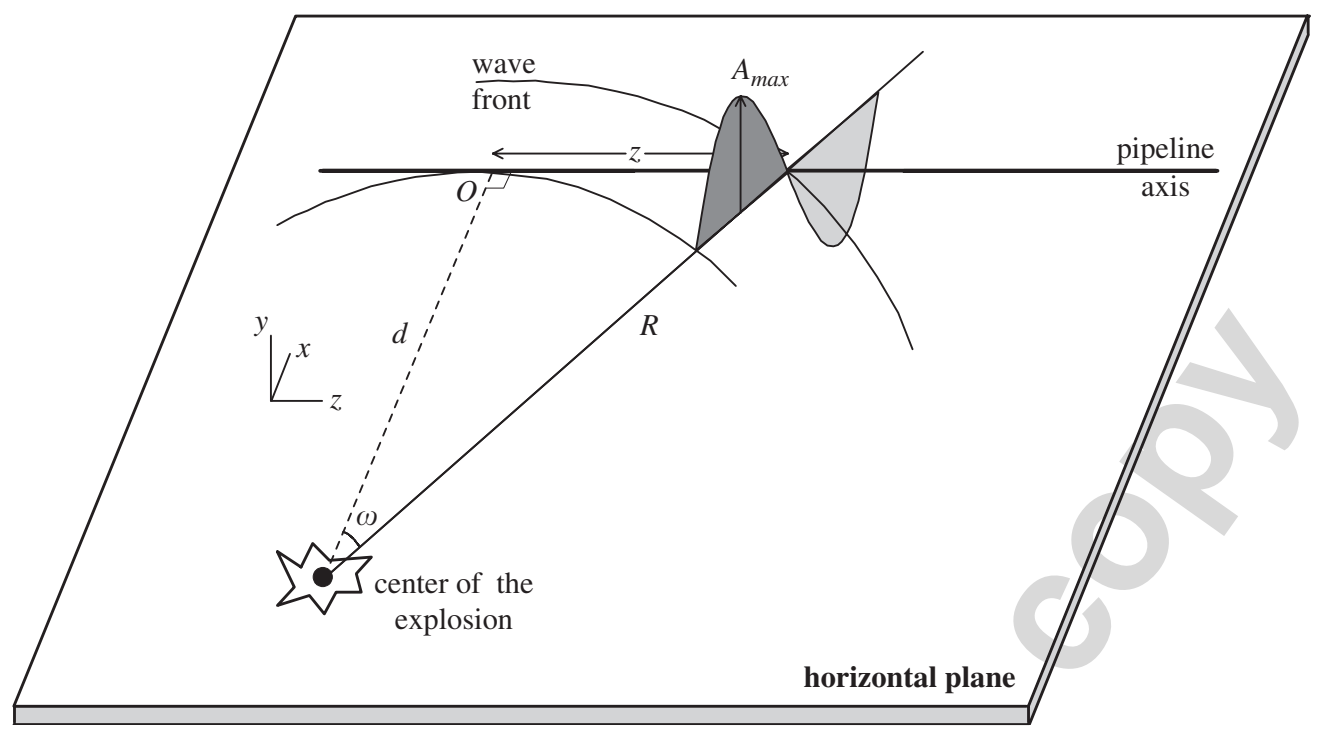

Fig. 7. Definition of the peak particle displacement $A_{\max }$ induced to the pipeline as a result of the radial propagation of the SV component of a blast-induced Rayleigh wave.

In a cylindrical coordinate system fitted to the axis of the structure (Fig. 4b), the above ground displacement is decomposed into the following radial and tangential components:

$$
\begin{aligned}
& u_{r}=A\left(\frac{R}{d+r \sin \theta}\right)^{-n} \cos \theta \sin \left[\frac{2 \pi}{L}\left(R^{\prime}-C_{R} t\right)\right], \\
& u_{\theta}=-A\left(\frac{R}{d+r \sin \theta}\right)^{-n} \sin \theta \sin \left[\frac{2 \pi}{L}\left(R^{\prime}-C_{R} t\right)\right] .
\end{aligned}
$$

The corresponding strains in the shell are consequently computed from thin-shell theory strain-displacement equations as

$$
\begin{aligned}
\varepsilon_{\alpha}= & \varepsilon_{z z}=\frac{\partial u_{z}}{\partial z}=0 \\
\varepsilon_{\mathrm{h}}= & \varepsilon_{\theta \theta}=\frac{1}{r} \frac{\partial u_{\theta}}{\partial \theta}+\frac{u_{r}}{r}=\frac{A}{2 d L R^{2}}(R / d)^{-n} \\
& \times \sin 2 \theta\left(2 d^{2} \pi R \cos \left[\frac{2 \pi}{L}\left(R-C_{R} t\right)\right]+L n z^{2} \sin \left[\frac{2 \pi}{L}\left(R-C_{R} t\right)\right]\right), \\
\gamma=\gamma_{\theta z}= & \frac{1}{r} \frac{\partial u_{z}}{\partial \theta}+\frac{\partial u_{\theta}}{\partial z}=\frac{A z}{L R^{2}}(R / d)^{-n} \\
& \times \sin \theta\left(2 \pi R \cos \left[\frac{2 \pi}{L}\left(R-C_{R} t\right)\right]-\operatorname{Ln} \sin \left[\frac{2 \pi}{L}\left(R-C_{R} t\right)\right]\right) .
\end{aligned}
$$

Again, to simplify the results, Eqs. (32) and (33) embrace the assumption that the radius of the pipeline $r$ is small compared to the distance from the explosion $d$ (Eq. (18)).

The complete set of analytical relations for strains induced by Rayleigh waves are derived by introducing the wave propagation velocity of Rayleigh waves $C_{R}$ to the strain equations for P- and SV waves, as well as a phase difference of $\pi / 2$ between the horizontal and the vertical component of ground motion. Thus, strains 
due to the compressional (P) Rayleigh wave component are

$$
\begin{aligned}
\varepsilon_{\alpha}= & \frac{A_{\max , \mathrm{H}}}{L R^{3}}(R / d)^{-n}\left(2 \pi z^{2} R \cos \left[\frac{2 \pi}{L}\left(R-C_{R} t\right)\right]\right. \\
& \left.+L\left(d^{2}-n z^{2}\right) \sin \left[\frac{2 \pi}{L}\left(R-C_{R} t\right)\right]\right), \\
\gamma= & \frac{A_{\max , \mathrm{H}} z}{d L R^{3}}(R / d)^{-n} \cos \theta\left(4 d^{2} \pi R \cos \left[\frac{2 \pi}{L}\left(R-C_{R} t\right)\right]\right. \\
& \left.+L\left(-d^{2}(2+n)+n z^{2}\right) \sin \left[\frac{2 \pi}{L}\left(R-C_{R} t\right)\right]\right) \\
\varepsilon_{\mathrm{h}}= & \frac{A_{\max , \mathrm{H}}}{L R^{3}}(R / d)^{-n} \cos ^{2} \theta\left(2 d^{2} \pi R \cos \left[\frac{2 \pi}{L}\left(R-C_{R} t\right)\right]\right. \\
& \left.+L z^{2}(1+n) \sin \left[\frac{2 \pi}{L}\left(R-C_{R} t\right)\right]\right) .
\end{aligned}
$$

In addition, strains due to the shear (SV) Rayleigh wave component are

$$
\begin{aligned}
\gamma= & \frac{A_{\max , \mathrm{V} z}}{L R^{2}}(R / d)^{-n} \sin \theta\left(-2 \pi R \sin \left[\frac{2 \pi}{L}\left(R-C_{R} t\right)\right]-L n \cos \left[\frac{2 \pi}{L}\left(R-C_{R} t\right)\right]\right), \\
\varepsilon_{\mathrm{h}}= & \frac{A_{\max , \mathrm{V}}}{2 d L R^{2}}(R / d)^{-n} \sin 2 \theta\left(-2 d^{2} \pi R \sin \left[\frac{2 \pi}{L}\left(R-C_{R} t\right)\right]\right. \\
& \left.+L n z^{2} \cos \left[\frac{2 \pi}{L}\left(R-C_{R} t\right)\right]\right) .
\end{aligned}
$$

Total shear and hoop strains on the shell due to the propagation of a Rayleigh wave can be computed from the superposition of Eqs. ((35) and (37)) and ((36) and (38)), respectively:

$$
\begin{aligned}
\gamma= & \frac{A_{\max , \mathrm{V} z}}{L R^{2}}(R / d)^{-n}\left(\{(1 / k) 4 d \pi \cos \theta-L \cdot n \cdot \sin \theta\} \cos \left[\frac{2 \pi}{L}\left(R-C_{R} t\right)\right]\right. \\
& \left.+\left\{\frac{(1 / k) L\left(-d^{2}(2+n)+n z^{2}\right) \cos \theta-2 \pi d R^{2} \sin \theta}{d R}\right\} \sin \left[\frac{2 \pi}{L}\left(R-C_{R} t\right)\right]\right) \\
\varepsilon_{\mathrm{h}}= & \left.\left.\frac{A_{\max , \mathrm{V}}(R / d)^{-n}\left(\left\{\frac{(1 / k) 4 d^{3} \pi \cos ^{2} \theta+L \cdot n \cdot z^{2} \sin 2 \theta}{L R^{2}}\right\} \cos \left[\frac{2 \pi}{L}\left(R-C_{R} t\right)\right]\right.}{2 d}\right\} \sin \left[\frac{2 \pi}{L}\left(R-C_{R} t\right)\right]\right) \\
& +\left\{\frac{\left.(1 / k) L z^{2}(1+n) \cos ^{2} \theta-\pi d R^{2} \sin 2 \theta\right)}{R}\left(\frac{(1 / 2)}{R}\right)\right.
\end{aligned}
$$

where $k$ is taken from Eq. (26).

Comparison with numerical results: Results obtained by employing the proposed methodology for a Rayleigh wave are consequently compared with the results of a FEM analysis with computer code ANSYS [20]. The pipe model is the same as described above for $\mathrm{P}$ waves, but the displacement excitation applied to the nodes of the 3-D shell is now described by the following equations, referring to the global 

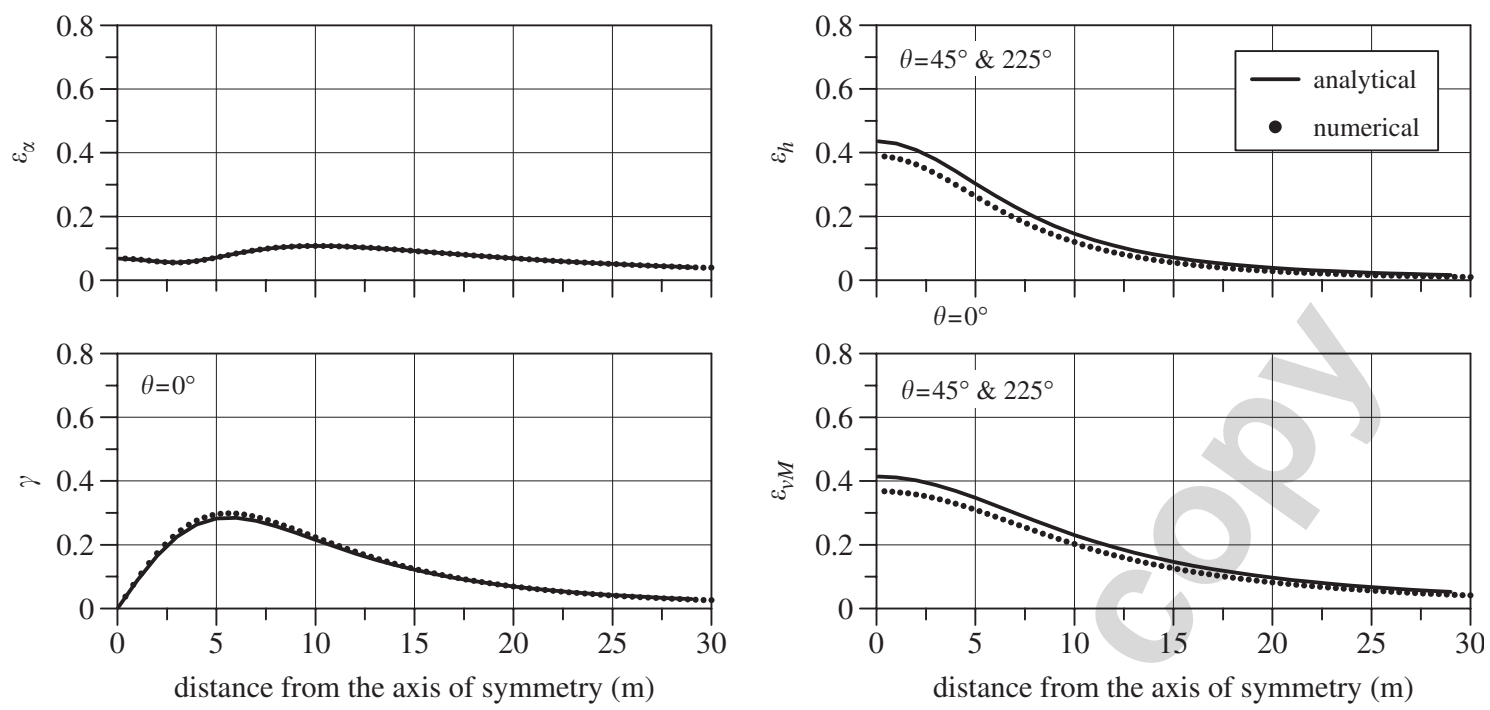

Fig. 8. Comparison of analytical and numerical results along the pipeline axis. The polar angle $\theta$ where each maximum strain appears is also shown (Rayleigh wave).

coordinate system of Fig. 5:

$$
\begin{aligned}
& u_{x, i}=\frac{1}{k} A\left(\frac{R_{i}}{x_{i}}\right)^{-n} \sin \omega_{i} \sin \left[\frac{2 \pi}{L}\left(R_{i}-C t\right)\right], \\
& u_{y, i}=A\left(\frac{R_{i}}{x_{i}}\right)^{-n} \sin \left[\frac{2 \pi}{L}\left(R_{i}-C t\right)+\pi / 2\right], \\
& u_{z, i}=\frac{1}{k} A\left(\frac{R_{i}}{x_{i}}\right)^{-n} \cos \omega_{i} \sin \left[\frac{2 \pi}{L}\left(R_{i}-C t\right)\right]
\end{aligned}
$$

and

$$
\theta_{x, i}=\theta_{y, i}=\theta_{z, i}=0 \quad \text { while } k=1.4677 .
$$

Numerical and analytical predictions of the maximum pipeline strains are compared in Fig. 8. The comparison is shown again in terms of the maximum overall (i.e. for the entire duration of the motion and over the cross-section) strain, although strains do not reach their maximum values along the pipeline at the same time instant and at the same polar angle $\theta$ (Fig. 8). Again, the analytical strains compare fairly well with the numerical results.

\section{Derivation of maximum strains along the pipeline}

The analytical expressions for the pipe strains derived in the above are functions, among others, of time $t$, a parameter that is not vital for the design of the pipeline. To conclude to a set of easy-to-use relations for design purposes, we must first evaluate the maximum, over time, strain in each cross-section, something that is accomplished with the procedure described briefly in the following.

To begin with, axial, hoop and shear strains are normalized against the $V_{\max } / C$ ratio, which is found to be the "reference" strain in many publications and guidelines referring to the design of buried pipelines against ground shock (e.g. [3,5,14]). The resulting normalized strains are mathematical expressions that quantitatively account for the effect of the spherical wave front, the attenuation of the peak particle velocity with the distance from the source, and the spatial and temporal superposition of strains (e.g. the innovations introduced by the presented methodology to account for the special characteristics of blast waves). 
The procedure for the derivation of the maximum, over time, normalized strains for each wave type and each strain component is a rather lengthy mathematical procedure. To limit the extent of the presentation, only the course of action for calculating the maximum normalized axial strain $\varepsilon_{\alpha, \text { max }}^{*}$ for $\mathrm{P}$ waves is presented in depth in the following.

Dividing Eq. (19) with the $\left(V_{\max } / C_{\mathrm{p}}\right)=\left(2 \pi A_{\max } / L\right)$ ratio yields the normalized axial strain:

$$
\varepsilon_{\alpha}^{*}=\frac{(R / d)^{-n}}{2 \pi R^{3}}\left(2 \pi z^{2} R \cos \left[\frac{2 \pi}{L}\left(R-C_{\mathrm{p}} t\right)\right]+L\left(d^{2}-n z^{2}\right) \sin \left[\frac{2 \pi}{L}\left(R-C_{\mathrm{p}} t\right)\right]\right) .
$$

Subsequently, the following mathematical treatment is adopted to reduce the number of independent variables: the distance $z$ from the projection of the center of the explosion measured along the axis of pipeline, and the distance from the center of the explosion to the pipeline axis $d$ (Fig. 3) are divided by the wavelength $L$ :

$$
\begin{aligned}
& z^{*}=z / L, \\
& d^{*}=d / L,
\end{aligned}
$$

while

$$
R^{*}=\sqrt{z^{* 2}+d^{* 2}} .
$$

In this way, 2 rather than 3 independent variables will appear in the mathematical expression for normalized strain. Substituting Eqs. (46) and (47) into Eq. (45) yields

$$
\varepsilon_{\alpha}^{*}=\left(R^{*} / d^{*}\right)^{-n}\left(\frac{z^{* 2}}{R^{* 2}} \cos \left[2 \pi\left(R^{*}-\frac{C_{\mathrm{p}} t}{T}\right)\right]+\frac{\left(d^{* 2}-n z^{* 2}\right)}{2 \pi R^{* 3}} \sin \left[2 \pi\left(R^{*}-\frac{C_{\mathrm{p}} t}{T}\right)\right]\right) .
$$

The maximization of Eq. (49) with respect to its temporal variables (that is the time $t$ and the wave propagation velocity $C_{\mathrm{p}}$ ) requires the computation of the time instant when $\varepsilon_{\alpha}^{*}$ becomes maximum along the pipeline axis. This is accomplished by algebraically solving the differential equation:

$$
\partial \varepsilon_{\alpha}^{*} / \partial t=0
$$

with the aid of the computer program Mathematica [22]. As $\varepsilon_{\alpha}^{*}$ is a harmonic function, Eq. (50) is satisfied by an infinite series of roots that have a phase difference of $2 \pi$. Those roots correspond to the time instances when the axial strain acquires its maximum value in a cross-section defined by $z^{*}$. Here, we are interested in the sequentially first one of the series of solutions, which represents the first time instant that the axial strain will become maximum. From those first four (4) feasible roots of Eq. (50):

$$
t=T R^{*} \pm \frac{T}{2 \pi} \arccos \left( \pm \frac{2 \pi z^{* 2} R^{*}}{\sqrt{\left(d^{* 2}-n z^{* 2}\right)^{2}+4 \pi^{2} z^{* 4} R^{* 2}}}\right)
$$

we keep the one that when substituted in Eq. (49) will provide the maximum $\varepsilon_{\alpha}^{*}$ value in each cross-section of the pipeline, through the entire duration of the ground shock, namely,

$$
\varepsilon_{\alpha, \max }^{*}=\frac{\left(R^{*} / d^{*}\right)^{-n}\left(4 \pi^{2} z^{* 4} R^{* 2}+\left(d^{* 2}-n z^{* 2}\right) \sqrt{\left(d^{* 2}-n z^{* 2}\right)^{2}}\right)}{2 \pi R^{* 3} \sqrt{4 \pi^{2} z^{* 4} R^{* 2}+\left(d^{* 2}-n z^{* 2}\right)^{2}}} .
$$

The same general concept is employed in the computation of normalized maximum shear and hoop strains, for both P- and Rayleigh waves. However, the latter strain components do not retain a uniform value along the cross-section as the axial strain does, but appear to be functions of the polar angle $\theta$ too (Eqs. (16), (17) and (39), (40)). As the introduction of the extra independent variable $\theta$ would make the abovementioned mathematical computation cumbersome, an approximate procedure is applied here: shear and hoop strains are computed at 8 characteristic points of the cross-section, with polar angles $\theta$ spaced at $\pi / 4$ (Fig. 9), and the highest among these 8 values are considered to be the maximum shear or hoop strain in the cross-section. 


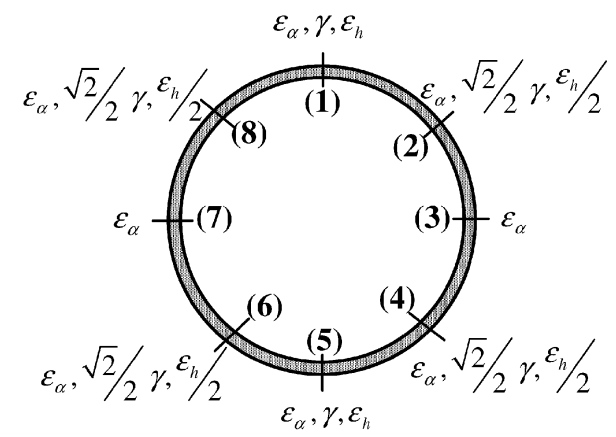

(a)

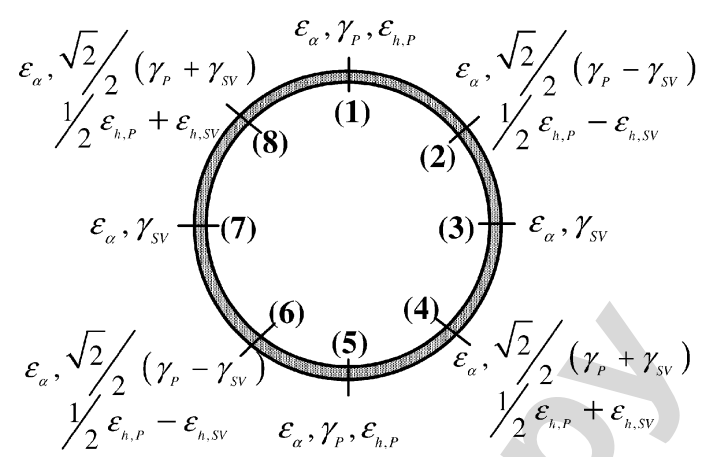

(b)

Fig. 9. Total strains at eight equally spaced points of the cross-section for (a) $\mathrm{P}$ waves and (b) Rayleigh waves. The subscripts $P$ and SV identify whether the specific strain component is due to the compressional or the shear component of the Rayleigh wave.

Table 1

Maximum normalized strains for $\mathrm{P}$ waves

\begin{tabular}{ll}
\hline Strain component & $\varepsilon_{\max }^{*}$ \\
\hline Axial & $\frac{\left(R^{*} / d^{*}\right)^{-n}\left(4 \pi^{2} z^{* 4} R^{* 2}+\left(d^{* 2}-n z^{* 2}\right) \sqrt{\left(d^{* 2}-n z^{* 2}\right)^{2}}\right)}{2 \pi R^{* 3} \sqrt{4 \pi^{2} z^{* 4} R^{* 2}+\left(d^{* 2}-n z^{* 2}\right)^{2}}}$ \\
Shear & $\frac{\left(R^{*} / d^{*}\right)^{-n} z^{*}\left(16 \pi^{2} d^{* 4} R^{* 2}+\left(d^{* 2}(2+n)-n z^{* 2}\right) \sqrt{\left(d^{* 2}(2+n)-n z^{* 2}\right)^{2}}\right)}{2 \pi d^{*} R^{* 3} \sqrt{16 \pi^{2} d^{* 4} R^{* 2}+\left(d^{* 2}(2+n)-n z^{* 2}\right)^{2}}}$ \\
& $\frac{\left(R^{*} / d^{*}\right)^{-n}}{2 \pi R^{* 3}} \sqrt{4 \pi^{2} d^{* 4} R^{* 2}+z^{* 4}(1+n)^{2}}$ \\
Hoop &
\end{tabular}

This procedure may appear pointless in the case of $\mathrm{P}$ waves, as in Fig. 9a we notice that strain maxima cannot appear but at points 1 and 5. In the case of Rayleigh waves though (Fig. 9b), the polar angles where shear and hoop strains become maximum appear to be functions of $z^{*}, d^{*}$ and $n$ too. For that, we compute $\gamma_{\max }^{*}$ and $\varepsilon_{\mathrm{h}, \max }^{*}$ at the 8 characteristic points on the cross-section, and locate the maximum one for design purposes on a case-by-case basis.

The expressions for each normalized maximum strain component due to $\mathrm{P}$ wave action are presented in Table 1 and drawn in Fig. 10 for a range of their three independent variables $\left(0.1 \leqslant z^{*} \leqslant 100,1 \leqslant d^{*} \leqslant 50\right.$, $n=1,2,3)$, illustrating only the symmetric half of the pipeline, with respect to the normal from the center of the explosion to the pipeline axis. With the aid of Fig. 10 we can compute not only the maximum strain over the entire length of the pipeline, but also the point along its axis where this occurs.

For the design of certain types of buried pipes, it is also useful to know the combined von Mises strain $\left(\varepsilon_{\mathrm{vM}}\right)$ as well as the major and minor principal strains $\left(\varepsilon_{1}, 3\right)$. Considering the strain state that develops in a 3-D thin shell, the relations that provide the aforementioned combined strains can be simplified to

$$
\begin{aligned}
& \varepsilon_{\mathrm{vM}}=\frac{1}{1+v_{l}} \sqrt{\varepsilon_{\alpha}^{2}+\varepsilon_{\mathrm{h}}^{2}-\varepsilon_{\alpha} \varepsilon_{\mathrm{h}}+\frac{3}{4} \gamma^{2}}, \\
& \varepsilon_{1,3}=\frac{\varepsilon_{\alpha}+\varepsilon_{\mathrm{h}}}{2} \pm \sqrt{\left(\frac{\varepsilon_{\alpha}-\varepsilon_{\mathrm{h}}}{2}\right)^{2}+\left(\frac{\gamma}{2}\right)^{2}} .
\end{aligned}
$$



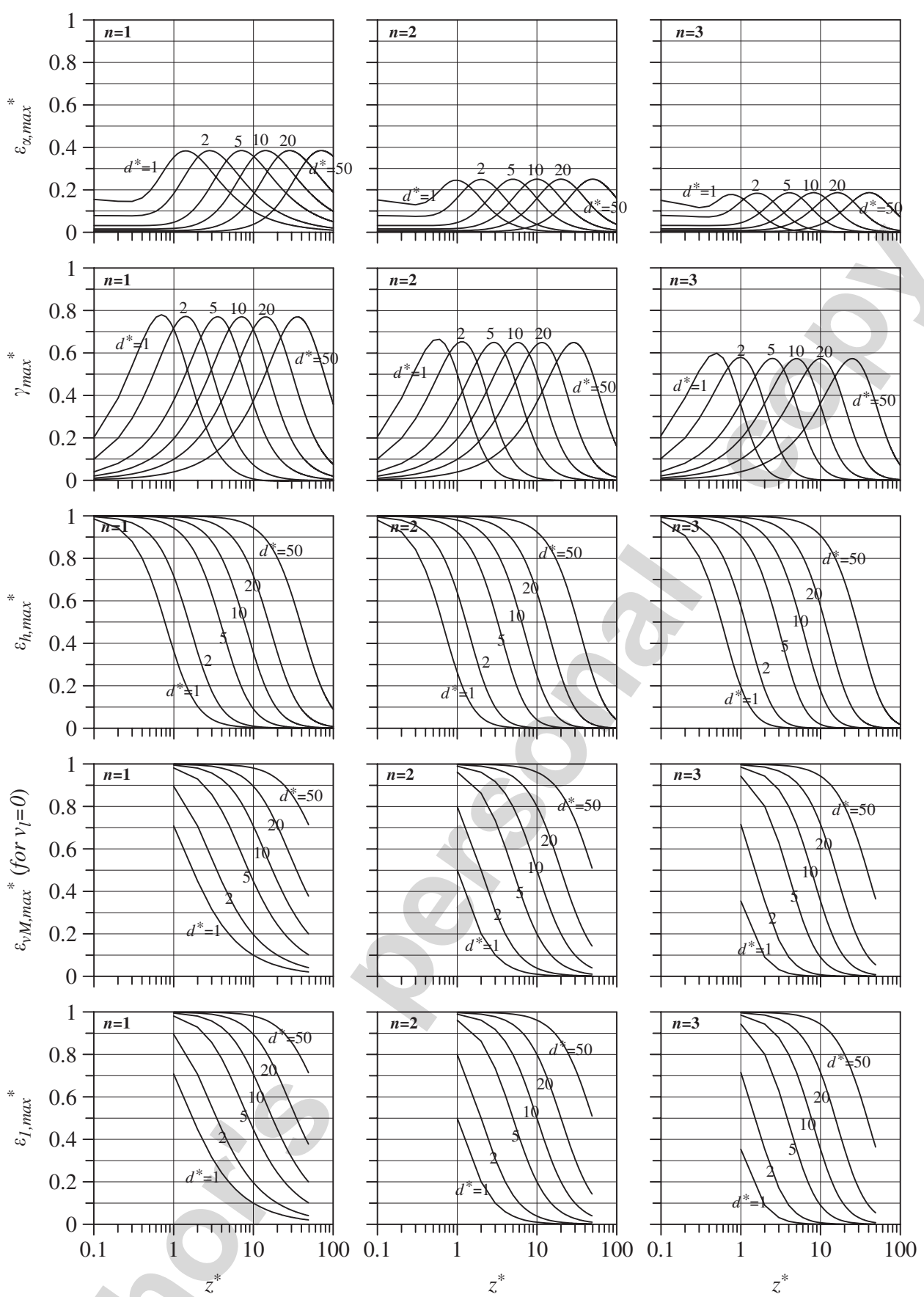

Fig. 10. Distribution of maximum (over time) normalized strains along the pipeline axis, for different ground attenuation exponents and distances from the explosion.

The complexity of Eqs. (53) and (54) (that is if we substitute the expressions for each strain component), makes the derivation of analytical expressions for the normalized von Mises and principal strains quite complicated. Still, by implementing the equations into a simple computer code, we can numerically calculate the maximum normalized von Mises and principal strains at the characteristic points of Fig. 9, by using a small space and time step for the superposition of their components. Plots for $\varepsilon_{\mathrm{vM} \text {,max }}^{*}$ and $\varepsilon_{1, \text { max }}^{*}$ computed this way for $\mathrm{P}$ waves are also presented in Fig. 10, for the same range of parameters used for the basic strain components. It must be noted that strains due to static loads (e.g. internal pressure) were overlooked in the computation of $\varepsilon_{\mathrm{vM} \text {,max }}^{*}$ and $\varepsilon_{1, \text { max }}^{*}$. If such strains are of the order of magnitude of blast-induced pipeline strains, Eqs. (53) and (54) must be properly re-written for the calculation of design von Mises and principal strains. 
To reduce the size of the presentation, the graphical representation of the maximum normalized strains for Rayleigh waves is omitted. However, the mathematical expressions that correspond to the total axial, shear and hoop strain at the characteristic points of Fig. $9 \mathrm{~b}$ are presented in Appendix A.

\section{Design relations}

The rather complicated analytical relations for the maximum normalized strains and the plethora of the required nomograms to substitute them make the calculation of maximum strains rather cumbersome for the design engineer. On the other hand, two important remarks, emerging from Fig. 10, can lead to further simplification of the proposed relations for design strains:

(a) the maximum normalized strain is not a function of the normalized distance $d^{*}$, and

(b) the position $z_{\max }^{*}$ along the axis of the pipeline where the maximum strain occurs appears to be independent, or in other cases, linearly dependent to the normalized distance $d^{*}$.

Bearing in mind the above, and using nomograms such as the ones shown in Fig. 10, we can express the maximum normalized strain over the entire length of the pipeline in terms of $n$, as

$$
\varepsilon_{\max }=\frac{V_{\max }}{C} \mathrm{CF}(n),
$$

where $\varepsilon_{\max }$ stands for the aforementioned maximum pipeline strains $\left(\varepsilon_{\alpha, \max }, \gamma_{\max }, \varepsilon_{\mathrm{h}, \max }, \varepsilon_{\mathrm{vM}, \max }, \varepsilon_{1, \max }\right)$, $C=C_{\mathrm{p}}$ for $\mathrm{P}$ waves and $C=C_{R}$ for Rayleigh waves, and $\mathrm{CF}(n)$ is the corresponding "Correction Factor". Plotting the correction factor for each strain component against the attenuation exponent $n$ (Fig. 11a for the $\mathrm{P}$ wave case) shows that a linear semi-logarithmic expression can be established for each correction factor, with the general form

$$
\mathrm{CF}(n)=-A \ln n+B \text {. }
$$

The exact expressions of $\mathrm{CF}(n)$ that should be used for the different components of pipeline strains are summarized in Tables 2 and 3, for P- and Rayleigh waves, respectively.

A similar expression applies to the position along the pipeline where the above maximum strains develop, defined in terms of the normalized distance $z_{\max } / d$ (Fig. 3), as shown graphically in Fig. $11 \mathrm{~b}$ for the $\mathrm{P}$ wave case, and expressed analytically in Tables 2 and 3.

Note that all but the axial, maximum strain components due to Rayleigh waves may develop at different locations on the pipeline cross-section, depending on the value of the attenuation exponent $n$. Yet, for typical
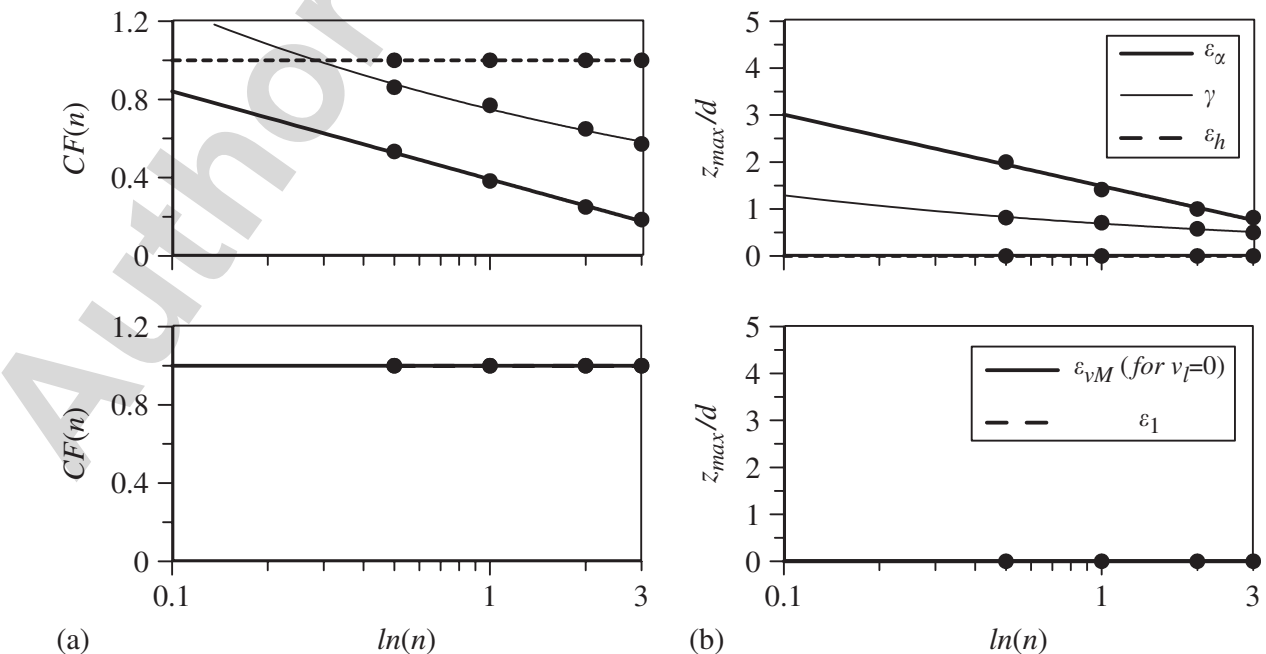

Fig. 11. Correction factors of the various strain components and their position $z_{\max }$ along the pipeline, plotted against the attenuation exponent $n-\mathrm{P}$ wave. 
Table 2

Simplified expressions for the calculation of the Correction Factor of each strain component and the position of the maximum along the pipeline axis-P wave

\begin{tabular}{lll}
\hline Strain component & $C F(n)$ & $z_{\max } / d$ \\
\hline Axial & $-0.195 \ln n+0.392$ & $-0.66 \ln n+1.489$ \\
Shear & $-0.162 \ln n+0.758$ & $-0.177 \ln n+0.7$ \\
Hoop & 1 & 0 \\
Von Mises & $1 /\left(1+v_{1}\right)$ & 0 \\
Principal & \pm 1 & 0 \\
\hline
\end{tabular}

Table 3

Simplified expressions for the calculation of the Correction Factor of each strain component and the position of the maximum along the pipeline axis-Rayleigh wave (for $k=1.4677$ )

\begin{tabular}{|c|c|c|}
\hline Strain component & $\mathrm{CF}(n)$ & $z_{\max } / d$ \\
\hline Axial & $-0.133 \ln n+0.267$ & $-0.661 \ln n+1.489$ \\
\hline Shear-position $1^{\mathrm{a}}$ & $-0.11 \ln n+0.516$ & $-0.176 \ln n+0.697$ \\
\hline Shear-positions 2 and $4^{\mathrm{a}}$ & $-0.127 \ln n+0.498$ & $-0.275 \ln n+0.82$ \\
\hline Shear-position $3^{\mathrm{a}}$ & $-0.165 \ln n+0.503$ & $-0.469 \ln n+1.052$ \\
\hline Hoop-position $1^{\mathrm{a}}$ & 0.681 & 0 \\
\hline Hoop-positions 2 and $4^{a}$ & 0.694 & 0 \\
\hline Hoop-position $3^{\mathrm{a}}$ & 0 & - \\
\hline Von Mises-position $1^{\mathrm{a}}$ & $0.681 /\left(1+v_{1}\right)$ & 0 \\
\hline Von Mises-positions 2 and $4^{\mathrm{a}}$ & $0.694 /\left(1+v_{1}\right)$ & 0 \\
\hline Von Mises-position $3^{\mathrm{a}}$ & $(-0.143 \ln n+0.435) /\left(1+v_{1}\right)$ & $-0.456 \ln n+1.052 /\left(1+v_{1}\right)$ \\
\hline Principal-position $1^{\mathrm{a}}$ & \pm 0.681 & 0 \\
\hline Principal-positions $2 \& 4^{\mathrm{a}}$ & \pm 0.694 & 0 \\
\hline Principal-position $3^{\mathrm{a}}$ & $\mp 0.115 \ln n \pm 0.295$ & $-0.456 \ln n+1.052$ \\
\hline
\end{tabular}

${ }^{\text {a }}$ Positions 1-4 refer to the cross-section of the pipeline and are defined in Fig. 9b.

ground conditions with $1.5<n<3.0$, the maximum shear strain occurs at point 1 of the cross-section (Fig. 9), while the maximum hoop, von Mises and major principal strains at points $2 \& 4$. The corresponding $\mathrm{CF}(n)$ and $z_{\max } / d$ relations are shade-marked in Table 3 .

\section{Comparison with field tests}

Validation of the proposed methodology is attempted through comparison to actual pipeline strain measurements due to full scale blasts. The aforementioned measurements were preformed by the US Bureau of Mines and the State of Indiana in cooperation with AMAX Coal Co. and its consultants, to monitor the effects of coal mine overburden blasting on nearby pipelines [6].

Results from 29 blasts of up to $950 \mathrm{~kg}$ per delay are shown in Figs. 12a and b, in terms of measured axial and hoop strains in 2 of the tested buried pipelines: one $50.8 \mathrm{~cm}$ diameter, $6.63 \mathrm{~mm}$ wall thickness steel pipeline and one $21.9 \mathrm{~cm}$ PVC pipeline with $8.43 \mathrm{~mm}$ wall thickness, located at distances from 20 to $1064 \mathrm{~m}$ from the nearest blast source of each test. These 2 pipelines were selected out of a total of 5 pipelines installed during the test blasts, since they are the only ones for which the flexibility criterion (Eq. (3)) is met, as noted by Siskind et al. [6]. Local soil conditions consist of a 12-m deep shale layer, covered with a 2-m layer of clayley soil. The pipelines were buried under $1 \mathrm{~m}$ of excavated clayley soil, while the depth of detonation varied from 13 to $20 \mathrm{~m}$.

The proposed analytical expressions for the calculation of axial and hoop strains (Eqs. (34) and (40), respectively) due to Rayleigh waves were employed for each blast test, as more appropriate for the distances considered. The case-specific attenuation relation derived by Siskind et al. [6] was used for the calculation of 

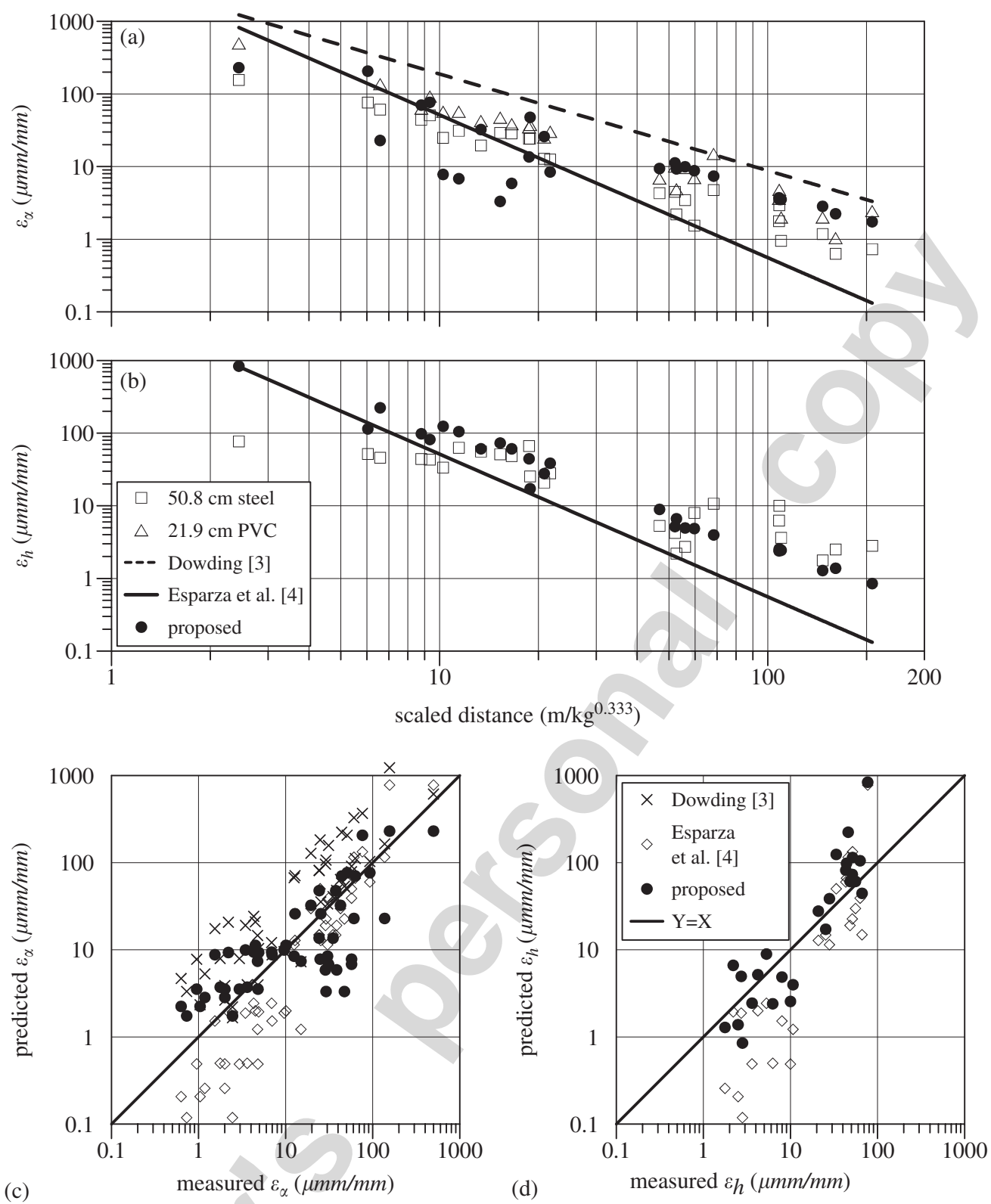

Fig. 12. Comparison of the results of the proposed methodology and of the current state-of-practice with field strain measurements presented by Siskind et al. [6].

the peak particle velocity at the position of the pipeline, namely,

$$
V_{\max }(\mathrm{m} / \mathrm{s})=3.22\left(\frac{R}{W^{0.333}}\right)^{-1.33},
$$

where $R$ is the distance from the nearest blast hole (in $\mathrm{m}$ ) and $W$ is the charge per delay (in $\mathrm{kg}$ ). The peak particle displacement $A_{\max }$ was calculated from the peak particle velocity using the following expression, valid for harmonic waves:

$$
A_{\max }=\frac{L_{R}}{2 \pi}\left(\frac{V_{\max }}{C_{R}}\right),
$$

where the Rayleigh wave propagation in shale, $C_{R}$, was taken to be equal to $800 \mathrm{~m} / \mathrm{s}$, while the wavelength $L_{R}$, was considered equal to $40 \mathrm{~m}$, based on the mean frequency content of the strong motion recordings reported by Sisking et al. [6]. 
Axial and hoop strains measured in the field and calculated by the proposed methodology are drawn in Figs. 12a and b, respectively, against the scaled distance of the pipeline from the nearest source blast, while their correlation is illustrated in Figs. 12c and d, in an one-on-one comparison.

In addition to the proposed methodology, strains on the pipelines are computed using the empirical relation proposed by Esparza et al. [4] for single-point source, as well as the simplified expression adopted by Dowding [3]. The exact relations and the input data used for these parallel predictions are given in Appendix B.

From Fig. 12 it is observed that results of the proposed methodology are generally compatible with the experimental measurements. On the other hand, the expression adopted by Dowding [3] provides an upper bound of measured axial strains, while the empirical expression of Esparza et al. [4] provides reasonably accurate results for relatively small-scaled distances $\left(<20 \mathrm{~m} / \mathrm{kg}^{0.333}\right)$, but underestimates axial and hoop strains thereafter.

To further investigate the potential effect of local soil conditions on pipeline strains, let us consider the fictitious case where the $50.8 \mathrm{~cm}$ steel pipeline considered in Siskind et al. [6] experiments is constructed near the surface of a wet clay layer, with $C_{\mathrm{s}}=250 \mathrm{~m} / \mathrm{s}$, and is subjected to a series of point source detonations of $1000 \mathrm{~kg}$ TNT in distances of 5, 10, 20 and $100 \mathrm{~m}$ from the pipeline.

Calculation of pipeline strains with all three methodologies follows the same workflow as described above. However, attenuation of the peak particle ground velocity in this case is much slower compared to the soft rock formation encountered in Siskind et al. experiments. Namely, for detonation of $1000 \mathrm{~kg}$ of TNT in wet clay the peak particle velocity is computed as [11]

$$
V_{\max }(\mathrm{m} / \mathrm{s})=16.08\left(\frac{R(m)}{730^{0.333}}\right)^{-1.35} \text {. }
$$

The comparison of predicted axial pipeline strains is shown in Fig. 13. Observe that the expression proposed by Esparza et al. [4] underestimates the maximum strains, for the whole range of distances of the pipeline from the explosion source. This is expectable, as the measurements on which the Esparza et al. empirical methodology is based on were conducted in a rock site, similar to that of the Siskind et al. [6] experiments, where the ground shock amplitude levels are considerably lower than in the wet clay. For example, at $20 \mathrm{~m}$ from the explosion source the peak particle velocity would reach $1.11 \mathrm{~m} / \mathrm{s}$ in the soft rock site (Eq. (57)) and $5.45 \mathrm{~m} / \mathrm{s}$ in the wet clay (Eq. (59)), exhibiting a five-fold increase.

On the contrary, Dowding's approximate method [3] provides, as in the comparison with real field data, over-conservative results. For example, axial strains computed with the proposed methodology are about 4.4 times smaller than these computed according to Dowding [3]. Taking into account that the peak particle

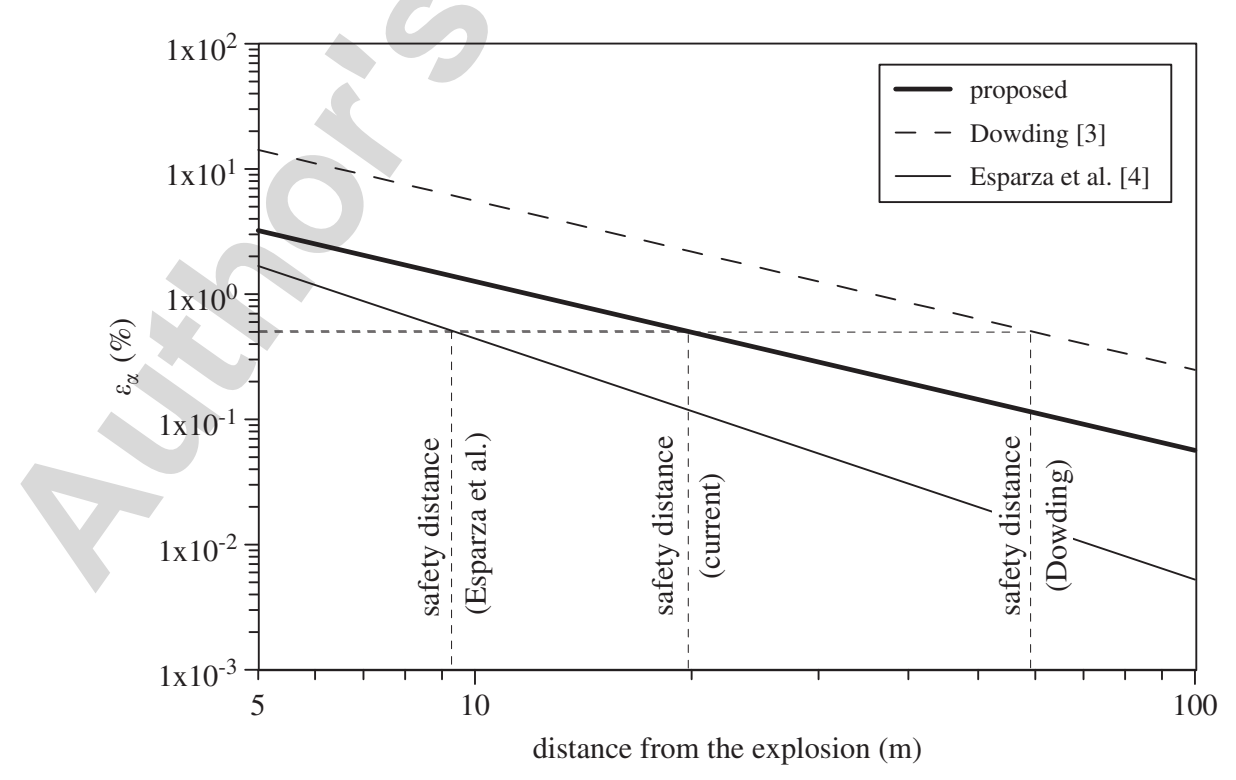

Fig. 13. Comparison of the results of the proposed methodology and of the current state-of-practice for the fictitious case of a $50.8 \mathrm{~cm}$ steel pipeline constructed in wet clay. 
velocity in both sets of predictions is calculated by the same expression (Eq. (59)), it may be concluded that the observed divergences are attributed to the fact that Dowding's analytical expression for axial strain does not account for the wave spherical front.

A feeling of how important such differences may become is obtained when the different methodologies are used to back-calculate the safety distance of the pipeline from the presumed explosion (Fig. 13). Supposing that the steel pipeline under consideration is constructed with peripheral in situ welds, the maximum axial strain on the cross-section should not exceed $0.5 \%$. For that strain limit, the safety distance to meet this requirement according to the results of the proposed methodology is $20 \mathrm{~m}$, while it increases to $60 \mathrm{~m}$, i.e. a three-fold increase, when Dowding's expression is adopted (Fig. 13).

\section{Summary and conclusions}

An analytical methodology to calculate blast-induced strains in buried pipelines has been presented, employing 3-D thin elastic shell theory in the analysis of the structure. Furthermore, compared to existing analytical and empirical methods, the proposed one takes consistently into account the special characteristics of blast-induced ground shock waves i.e. the spherical front and the soil-dependant exponential attenuation of their amplitude with the distance from the source. Simple design relations are provided to aid the practical application of the method.

The proposed relations have been evaluated against field measurements of blast-induced axial and hoop pipeline strains reported in the literature. In addition, a thorough comparison was made with two methods frequently used in current practice: these of Dowding [3] and Esparza et al. [4]. In conclusion, it was found that the proposed method has achieved improved accuracy at no major expense of simplicity. In comparison, Dowding's methodology, which was originally developed for seismic waves with a plane instead of a spherical front, provides consistently higher estimates of pipeline strains. On the other hand, the empirical methodology of Esparza et al. provides comparable predictions for relatively short distances from the blast and stiff-dry ground conditions, simulating the conditions prevailed in the field experiments which were used to calibrate the method. Nevertheless, it may considerably underestimate pipeline strains for longer distances, or wet soil conditions where the ground shock attenuation becomes slower.

\section{Appendix A. Maximum normalized strains for Rayleigh waves}

Axial strain:

$$
p \frac{\left(R^{*} / d^{*}\right)^{-n}\left(4 \pi^{2} z^{* 4} R^{* 2}+\left(d^{* 2}-n z^{* 2}\right) \sqrt{\left(d^{* 2}-n z^{* 2}\right)^{2}}\right)}{2 \pi R^{* 3} \sqrt{4 \pi^{2} z^{* 4} R^{* 2}+\left(d^{* 2}-n z^{* 2}\right)^{2}}} .
$$

Shear strain-position (1):

$$
p \frac{\left(R^{*} / d^{*}\right)^{-n} z^{*}\left(16 \pi^{2} d^{* 4} R^{* 2}+\left(d^{* 2}(2+n)-n z^{* 2}\right) \sqrt{\left(d^{* 2}(2+n)-n z^{* 2}\right)^{2}}\right)}{2 \pi d^{*} R^{* 3} \sqrt{16 \pi^{2} d^{* 4} R^{* 2}+\left(d^{* 2}(2+n)-n z^{* 2}\right)^{2}}} .
$$

Shear strain-position (2):

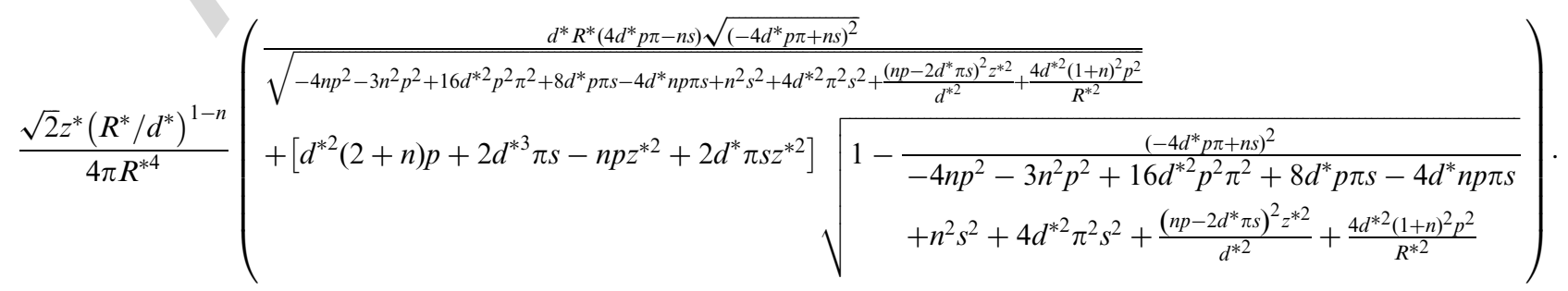


Shear strain-position (3):

$$
s \frac{\left(R^{*} / d^{*}\right)^{-n} z^{*}}{2 \pi R^{* 2}} \sqrt{n^{2}+4 \pi^{2} R^{* 2}}
$$

Shear stain-position (4):

$$
\frac{\sqrt{2} z^{*}\left(R^{*} / d^{*}\right)^{1-n}}{4 \pi R^{* 4}}\left(\begin{array}{l}
\frac{d^{*} R^{*}\left(4 d^{*} p \pi+n s\right) \sqrt{\left(4 d^{*} p \pi+n s\right)^{2}}}{\sqrt{-4 n p^{2}-3 n^{2} p^{2}+16 d^{* 2} p^{2} \pi^{2}-8 d^{*} p \pi s+4 d^{*} n p \pi s+n^{2} s^{2}+4 d^{* 2} \pi^{2} s^{2}+\frac{\left(n p+2 d^{*} \pi s\right)^{2} z^{* 2}}{d^{* 2}}+\frac{4 d^{* 2}(1+n)^{2} p^{2}}{R^{* 2}}}} \\
+\left[-d^{* 2}(2+n) p+2 d^{* 3} \pi s+n p z^{* 2}+2 d^{*} \pi s z^{* 2}\right] \\
\begin{array}{c}
1-\frac{\left(4 d^{*} p \pi+n s\right)^{2}}{-4 n p^{2}-3 n^{2} p^{2}+16 d^{* 2} p^{2} \pi^{2}-8 d^{*} p \pi s+4 d^{*} n p \pi s+n^{2} s^{2}} \\
+4 d^{* 2} \pi^{2} s^{2}+\frac{\left(n p+2 d^{*} \pi s\right)^{2} z^{* 2}}{d^{* 2}}+\frac{4 d^{* 2}(1+n)^{2} p^{2}}{R^{* 2}}
\end{array}
\end{array} .\right.
$$

Hoop strain-position (1):

$$
p \frac{\left(R^{*} / d^{*}\right)^{-n}}{2 \pi R^{* 3}} \sqrt{4 \pi^{2} d^{* 4} R^{* 2}+z^{* 4}(1+n)^{2}}
$$

Hoop strain-position (2):

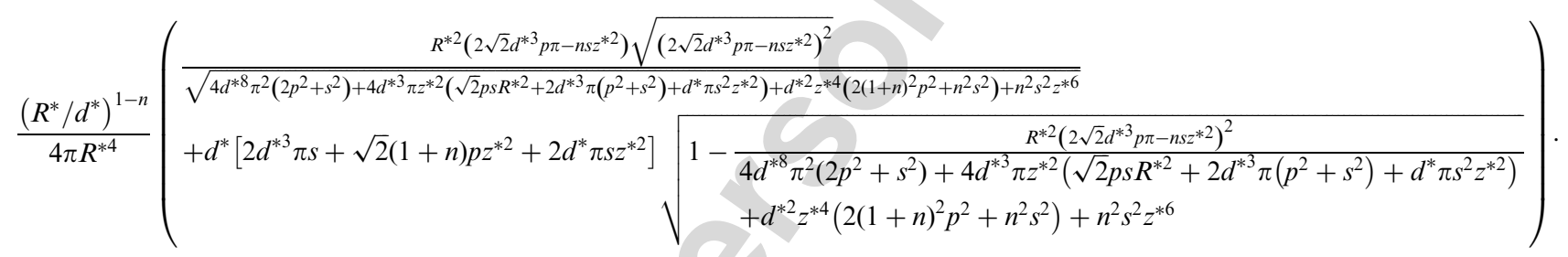

Hoop strain-position (4):

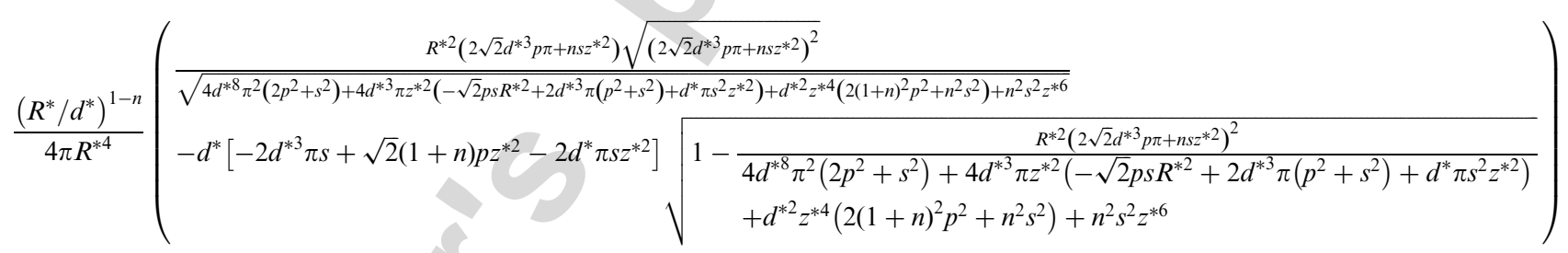

(1): $p=A_{\max , \mathrm{H}} / A_{\max }$ and $s=A_{\max , \mathrm{V}} / A_{\max }(2)$ : positions $1-4$ refer to the cross-section of the pipeline and are defined in Fig. $9 \mathrm{~b}$

\section{Appendix B. Prediction of field-measured strains using the current design practice}

Calculation of strains using the methodology proposed by Esparza et al. [4]: Esparza et al. [4] suggest that axial and hoop stresses on steel pipelines due to nearby blasts can be computed using the following empirical relation, derived from the statistical evaluation of a series of field tests for single and multi-shot blasts:

$$
\sigma=4.44 E_{1}\left(\frac{K_{4} W_{\text {eff }}}{\sqrt{E_{1} t_{\mathrm{s}}} R^{K_{5}}}\right)^{K_{6}},
$$

where $E_{1}$ is the Young's modulus of steel (in psf), $t_{\mathrm{s}}$ the pipe wall thickness (in $\mathrm{ft}$ ), $W_{\text {eff }}$ the effective weight of the explosives (in pounds), accounting for the orientation of the explosives relatively to the pipeline, $R$ the distance between the pipe and the explosives, grater than 2 pipe diameters, and $K_{i}$ empirical coefficients. 
The problem examined herein, due to the delayed-type blasts, corresponds to the single-point source case of Esparza et al.'s relation, as also remarked by Siskind et al. [6], so Eq. (B.1) becomes

$$
\sigma=4.44 \times 4.393 \times 10^{9} \mathrm{psf}\left(\frac{1(0.98 \times 2207.5 \text { pounds })}{\sqrt{4.393 \times 10^{9} \mathrm{psf} \times 0.0328 \mathrm{ft}}[R(\mathrm{ft})]^{2.5}}\right)^{0.77} .
$$

The strains corresponding to the stresses computed from Eq. (B.2) are presented in Figs. 12a and b, and are correlated to measured strains in Figs. $12 \mathrm{c}$ and d, next to results of the proposed methodology.

Calculation of strains using the methodology proposed by Dowding [3]: For the calculation of blast-induced axial strains Dowding [3] adopts the following approximate expression, originally proposed for the seismic verification of buried pipelines and tunnels [1]:

$$
\varepsilon_{\alpha}=V_{\max } / C
$$

where $V_{\max }$ is calculated from Eq. (57) for various distances from the explosion and $C=800 \mathrm{~m} / \mathrm{s}$. Axial strains calculated from Eq. (B.3) are also presented in Fig. 12a and compared to the measured axial strains in Fig. 12c.

\section{References}

[1] Newmark NM. Problems in wave propagation in soil and rock. In: Proceedings of the International Symposium on Wave Propagation and Dynamic Properties of Earth Materials. Albuquerque, NM: University of New Mexico Press; August 23-25, 1968. p. 7-26.

[2] Kuesel TR. Earthquake design criteria for subways. J Struct Div ASCE 1969;ST6:1213-31.

[3] Dowding CH. Blast vibration monitoring and control. Englewood Cliffs, NJ: Prentice-Hall Inc.; 1985.

[4] Esparza ED, Westine PS, Wenzel AB. Pipeline response to buried explosive detonations, vol. 1. Southwest Research Institute Report to the American Gas Association, AGA Project, PR-15-109, 1981.

[5] American Lifelines Alliance. Guidelines for the design of buried steel pipes. New York: ASCE; July 2001.

[6] Siskind DE, Stagg MS, Wiegand JE, Schulz DL. Surface mine blasting near pressurized transmission pipelines. Report of Investigations RI9523, to US Department of Interior, Bureau of Mines, 1994.

[7] Lamb H. On the propagation of tremors over the surface of an elastic solid. Philos Trans R Soc London Ser A 1904(203):1-42.

[8] Mooney HM. Some numerical predictions for Lamb's problem. Bull Seismol Soc Am 1974;64:473.

[9] Samoreli DG. Elasto-plastic analysis of the propagation of blast waves into the ground. Diploma thesis, Geotechnical Division, Faculty of Civil Engineering, NTUA, 1998 [in Greek].

[10] $\mathrm{Wu} \mathrm{C}$, Hao $\mathrm{H}$. Modeling of simultaneous ground shock and airblast pressure on nearby structures from surface explosions. Int $\mathbf{J}$ Impact Eng 2005;31:699-717.

[11] TM5-855-1. Design and analysis of hardened structures to conventional weapons effects. The Departments of Army, Air Force and Navy and the Defence Special Weapons Agency, USA, 1998.

[12] Negmatullaev SKh, Todorovska MI, Trifunac MD. Simulation of strong earthquake motion by explosions-experiments at the Lyaur testing range in Tajikistan. Soil Dyn Earthquake Eng 1999;18:189-207.

[13] Henrych J. The dynamics of explosion and its use. Amsterdam: Elsevier; 1979.

[14] European Committee for Standardisation. Eurocode 8: Design of structures for earthquake resistance. Part 4-Silos, Tanks and Pipelines. Draft No. 2, September 2003.

[15] Hoeg K. Stresses against underground structural cylinders. J Soil Mech Found Div ASCE 1968;94, SM4:833-58.

[16] Hendron Jr AJ, Fernandez G. Dynamic and static considerations for underground chambers. Seismic design of embankments and caverns. New York: ASCE; 1983. p. 157-197.

[17] O'Rourke MJ, Liu X. Response of buried pipelines subject to earthquake effects. Monograph Series MCEER 1999.

[18] Kelkar VS, Sewell RT. Fundamentals of the analysis and design of shell structures. Englewood Cliffs, NJ: Prentice-Hall Inc.; 1987.

[19] Kouretzis GP, Bouckovalas GD, Gantes CJ. 3-D shell analysis of cylindrical underground structures under seismic shear (S) wave action. Soil Dyn Earthquake Eng 2006;26:909-21.

[20] Ansys Inc. ANSYS 9.0 Documentation, 2004.

[21] Ewing WM, Jardesky WS, Press F. Elastic waves in layered media. New York: McGraw-Hill; 1957.

[22] Wolfram Research Inc. Mathematica 4.0 User's Manual. 1999. 